\title{
NUEVOS DATOS SOBRE ALGUNAS ESPECIES DE HEMERÓBIDOS (INSECTA, NEUROPTERA, HEMEROBIIDAE)
}

\author{
V. J. Monserrat ${ }^{1}$
}

\begin{abstract}
RESUMEN
Se anotan nuevos datos sobre la distribución, biología, fenología, morfología alar o genital, variabilidad, nomenclatura y / o taxonomía de 67 especies de hemeróbidos de las Faunas Paleártica, Neártica, Afrotropical, Oriental, Australiana y Neotropical. Alguna de ellas no había sido citada desde su descripción original y de otras se amplía significativamente su distribución.

Se anotan nuevos datos sobre la morfología y pigmentación de las alas de Hemerobius cercodes Navás, 1917, Sympherobius distinctus Carpenter, 1940, S. similis Carpenter, 1940 y S. limbus Carpenter, 1940 y sobre la genitalia externa masculina de Sympherobius limbus Carpenter, 1940, S. similis Carpenter, 1940, S. arizonicus Banks, 1911, S. perparvus (McLachlan, 1869), S. occidentalis (Fitch, 1855) y S. angustus (Banks, 1904).

Se propone la creación de un neotipo para Sympherobius pupillus Navás, 1915 y para Hemerobius cercodes Navás, 1917 y son propuestas dos nuevas sinonimias: Sympherobius angustus $($ Banks, 1904) $=$ S. pupillus Navás, 1915 n. sin. y Hemerobius cercodes Navás, $1917=$ H. subacutus $($ Nakahara, 1966) n. sin. En base a la gran variabilidad hallada en diferentes poblaciones/ ejemplares del grupo de especies neárticas de Sympherobius angustus (Banks, 1904) y debido a la falta de correlación y el solapamiento de caracteres utilizados para definirlas, se pone en duda la validez de algunas de las especies de este grupo. Una nueva especie Notiobiella jaimei n. sp. se describe de Vietnam.

Palabras clave: Insecta, Neuroptera, Hemerobiidae, faunística, biología, fenología, morfología, variabilidad, taxonomía, Paleártico, Neártico, Oriental, Afrotropical, Australiana, Neotropical.
\end{abstract}

\begin{abstract}
New data on some brown lacewing species (Insecta, Neuroptera, Hemerobiidae)

New data on the distribution, biology, phaenology, wing or genital morphology, variability, nomenclature and/or taxonomy of 67 brown-lacewings species from Palaearctic, Nearctic, Holarctic, Afrotropical, Oriental, Australian and Neotropical Faunas are given. New data about some species not recorded since the original description are given, and the previously known geographical distribution of some species is significantly enlarged.
\end{abstract}


New data on the wing pigmentation and morphology of Hemerobius cercodes Navás, 1917, Sympherobius distinctus Carpenter, 1940, S. similis Carpenter, 1940 and $S$. limbus Carpenter, 1940 are given, and data on the external male genitalia of Sympherobius limbus Carpenter, 1940, S. similis Carpenter, 1940, S. arizonicus Banks, 1911, S. perparvus (McLachlan, 1869), S. occidentalis (Fitch, 1855) and S. angustus (Banks, 1904) are figured.

Neotypes for Sympherobius pupillus Navás, 1915 and for Hemerobius cercodes Navás, 1917 are proposed, and two new synonymies: Sympherobius angustus (Banks, $1904)=S$. pupillus Navás, 1915 n. sin. and Hemerobius cercodes Navás, $1917=H$. subacutus (Nakahara, 1966) n. sin. are proposed. On the basis of the high variability and overlapping found in several external and genital characters that define some Nearctic species / populations of the Sympherobius angustus (Banks, 1904) species group, some species of this species group are questionable. A new species, Notiobiella jaimei $\mathbf{n}$. sp. is described from Vietnam.

Key words: Insecta, Neuroptera, Hemerobiidae, Faunistics, Biology, Phaenology, Morphology, Variability, Taxonomy, Palaearctic, Nearctic, Oriental, Afrotropical, Australian, Neotropical.

\section{Introducción}

Los hemeróbidos constituyen una interesante familia de neurópteros debido a su amplia distribución geográfica, al elevado número de individuos que frecuentemente constituyen sus poblaciones y especialmente por su utilización como agentes de control de pequeños artrópodos fitófagos (McEwen et al., 2001). No obstante, es proporcionalmente escasa la atención que vienen recibiendo en comparación con otras familias de neurópteros con interés en el control biológico como Chrysopidae o Coniopterygidae, y son proporcionalmente escasos los datos existentes sobre la distribución, la biología, fenología, morfología, estadios juveniles y variabilidad de la mayoría de las 600 especies que se consideran válidas en esta familia, muchas de las cuales aún poseen una situación taxonómica mal definida (Makarkin, 1990; Monserrat, 1990a, b, c, d, 1991, 1993, 1994, 2003; Monserrat \& Deretsky, 1999; Oswald, 1988, 1990, 1993a, b, 1994, 1996; Hölzel, 2007, etc.).

A nivel genérico, su taxonomía y sistemática ha sido recientemente tratada (Oswald, 1993a), sin embargo, su estado general de conocimiento es aún muy incompleto, siendo francamente escasos los trabajos de revisión global en la mayoría de sus géneros, son muchas las especies inadecuadamente descritas y tipificadas, otras no han sido citadas posteriormente desde su descripción original y otras son muy parcialmente conocidas, siendo muy parco el conocimiento sobre su morfología, venación, genitalia y variabilidad de muchas especies, hecho que conlleva a errores de identificación y que provoca confusiones en su distribución geográfica real, y en la mayoría de sus especies son desconocidos sus estadios larvarios y su biología. Por ello, de forma global, puede decirse que la situación taxonómica y sistemática de muchas de las especies de esta familia es bastante incompleta y fragmentaria.

Sin duda, las faunas Paleártica y Neártica son las mejor conocidas sobre este particular, si bien el límite real de distribución de muchas especies es, con frecuencia, impreciso o incompleto y, en ocasiones, los datos sobre su biología son escasos y fragmentarios y el nivel de conocimiento de las faunas de muchos países, incluso en zonas relativamente bien estudiadas como Europa y Estados Unidos es aún poco satisfactorio. Este hecho es aún más evidente en faunas de otros continentes y nuevos trabajos faunísticos van aportando nuevos datos que van completado las lagunas existentes sobre su distribución, biología o estadios juveniles (Klimaszewski \& Kevan, 1985, 1987a, b, 1988a, b, 1990a, b, c, 1992; Kevan \& Klimaszewski, 1986, 1987; Aspöck et al., 1980, 2001; Monserrat, 1980a, b, 1984, 1985, 1986a, 1990a, b, c, d, 1991, 1992a, b, 1993, 1994, 1996, 2000, 2001, 2003, 2004; New, 1988a, b; Oswald, 1988, 1990, 1993a, b, 1994, 1996; Makarkin, 1990, 1993; Marín \& Monserrat, 1995; Monserrat \& Marín, 1996; Monserrat \& Deretsky, 1999, Hölzel, 2007, etc.).

En el presente artículo anotamos nuevos datos sobre la nomenclatura, taxonomía, faunística, fenología, biología, morfología alar o genital y / o variabilidad de 67 especies que citamos de Europa, Asia, África, América y Australia.

\section{Material y método}

Se ha estudiado diverso material recolectado por el autor, así como material de las colecciones de varias Instituciones. Para indicar la ubicación y per- 
tenencia del material estudiado se emplean las siglas que se enumeran a continuación:

(FSCA) Florida Department of Agriculture (Gainesville, USA).

(HNHM) Hungarian Natural History Museum (Budapest, Hungría).

(IA) Insectario de Almazora, Servicio de Sanidad Vegetal (Almazora, Castellón, España).

(INHS) Illinois State Natural History Survey (Champaign, USA).

(MNCNM) Museo Nacional de Ciencias Naturales (Madrid, España).

(MZB) Museu de Zoologia (Barcelona, España).

(MZSP) Museu de Zoologia da Universidade de São Paulo (Brasil).

(NHM) The Natural History Museum (Londres, Reino Unido).

(PD) Colección de Peter Duelli, Swiss Federal Research Institute (Birmensdorf, Suiza).

(TAMU) Department of Entomology, Texas A. \& M. University (College Station, USA).

(TM) Transvaal Museum (Pretoria, Sudáfrica).

(UL) Universidad de León (León, España).

(ULL) Universidad de La Laguna (Tenerife, España).

(VM) Colección del autor, Universidad Complutense (Madrid, España).

El material estudiado se cita ordenado alfabética y cronológicamente siguiendo la siguiente secuencia máxima: País, provincia / departamento / estado, condado o isla, localidad de captura, coordenadas, altitud, fecha de captura, número de $0^{\top} \sigma^{\top}$ o de 우 estudiados, datos de biología, recolector e institución a la que dicho material pertenece, según las siglas anotadas. Se usa un asterisco $(*)$ para indicar los ejemplares utilizados en las figuras de sus alas $\left(\mathrm{A}^{*}\right)$ o su genitalia masculina $\left(\sigma^{\top *}\right)$.

Para acceder a la genitalia de los ejemplares conservados en seco se reblandecía el extremo del abdomen con agua durante unas horas y todos (conservados secos o en alcohol) se aclaraban con ácido láctico caliente. Ocasionalmente, se ha empleado negro de clorazenol para acceder a las estructuras internas menos visibles. Las fotografías se han realizado a través de lupa Motic SMZ-168 adaptada a cámara Canon Eos 300D digital.

De cada especie indicada se anota un pequeño comentario con su distribución general y datos de su biología, así como cualquier otro dato que merezca ser anotado. Para la terminología morfológica y genital se sigue a Tjeder (1961) y Aspöck et al. (1980) y para la ordenación sistemática se sigue a Oswald (1993a, b, 1994).

\section{Material estudiado}

\section{Hemerobiidae Latreille, 1802}

\section{Hemerobiinae Latreille}

\section{Hemerobius humulinus Linnaeus, 1758}

Elemento Holártico extendido en América desde Alaska a Guatemala y frecuente en las zonas boscosas y húmedas y citado sobre gran variedad de plantas.

Material estudiado: ESPAÑA: Asturias, Cangas de Onís, 5.VIII.2004, 1m sobre Corylus avellana, 1 ㅇ a la luz, V. J. Monserrat (VM). Guipúzcoa, Aiako Harria P. N., Bistarri, Hernani, 300 m, 30TWN8787, 17.IX.2002, 1 ㅇ sobre Alnus glutinosa, I. Zabalegui (VM), Irún, Río Bidasoa, $100 \mathrm{~m}$, 13.VI.2006, $10^{7}$ en ribera, P. Duelli (PD), Lareo, $750 \mathrm{~m}$, 30TWN7259, 16.VI.2005, 1 ㅇ sobre Betula alba, S. Pagola (VM). La Rioja, Haro, 475 m, 30TWN1315, 10.VI.2005, $10^{7}$ sobre Salix alba, S. Pagola (VM). Vizcaya, Larruskain, 106 m, 17.VI.2006, 1 ㅇ , P. Duelli (PD), Santa Lucía, $450 \mathrm{~m}$,

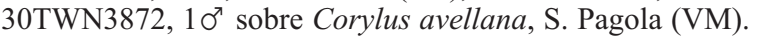

\section{Hemerobius nitidulus Fabricius, 1777}

Especie Paleártica occidental y central, asociada a coníferas.

Material estudiado: ESPAÑA: Álava, Ribera, 750-800 m, 30TVN8144, 12.VII.2005, $20^{7} 0^{7}$ sobre Pinus sylvestris, S. Pagola (VM). Barcelona, Borredá, 880 m, 31TDG16, 5.VIII.2005, 1 ㅇ, S. Pagola (VM). Madrid, El Escorial, Abantos, Fuente del Cervunal, 3.VII.2007, 2 우 $ᄋ$, 24.VII.2007, $60^{7} 0^{7}, 6$ 우 sobre Pinus sylvestris, V. J. Monserrat (VM).

\section{Hemerobius micans Olivier, 1792}

Elemento Paleártico occidental, generalmente asociado a planifolios en medios húmedos.

Material estudiado: ESPAÑA: Álava, S. Justi-Kapildui, Berdales, Iski P. N., 970 m, 30TWN3831, 14.VII.2004, 1 ㅇ, S. Pagola (VM). Guipúzcoa, Elosua, 520 m, 30TWN57, 9.VII.2005, $10^{7}$, I. Zabalegui (VM), Oieleku, Oiartzun, Aiako Harria P. N., 30TWN9589, 500-650 m, 2.VII.2006, $20^{7} 0^{7}$ sobre Corylus avellana, S. Pagola (VM), 25.VII-18.VIII.2006, $10^{7}$ sobre Fagus sylvati$c a$, S. Pagola (VM). Navarra, Baratxueta, 900 m, 13.VI.2006, 1 ㅇ sobre Fagus sylvatica, P. Duelli (PD), Hachueta, Santiago San Miguel, 1200 m, 15.VI.2006, $10^{7}$ sobre Fagus sylvatica, P. Duelli (PD). Zaragoza, Moncayo, Fuetne de la Teja, 7.VIII.2007, 3 우 sobre Fagus sylvatica, V. J. Monserrat (VM).

\section{Hemerobius contumax Tjeder,1932}

Especie conocida de Europa y Anatolia, asociada a coníferas (Larix, Pinus, Picea).

Material estudiado: ESPAÑA: Navarra, Portillo de Eraice, 1537 m, 18.VI.2006, $10^{\pi}$, P. Duelli (PD).

\section{Hemerobius stigma Stephens, 1836}

Especie holártica, mayoritariamente asociada a coníferas. 
Material estudiado: ESPAÑA: Álava, Ribera, 750-800 m, 30TVN8144, 12.VII.2005, $20^{7} 0^{7}, 1$ ㅇ sobre Pinus sylvestris, S. Pagola (VM). Castellón, Vistabella, San Juan de Peñagolosa, 8.II.2004, 1 ㅇ en trampa adhesiva con feromona sexual de Matsucoccus feytaudi sobre Pinus pinaster, M. E. Rodrigo (VM), Pina de Montalgrao, Santa Bárbara, 12.II.2004, 3 ㅇ o en trampa adhesiva con feromona sexual de Matsucoccus feytaudi sobre Pinus pinaster, M. E. Rodrigo (VM). Guipúzcoa, Buenabista, Oiatzun, 330 m, 30TWN9594, 21.VII.2004, $10^{n}$, S. Pagola (VM). Jaén, Collado de los Jardines, 20.VI.2004, 1 ㅇ sobre Juniperus oxycedrus, V. J. Monserrat (VM). León: León, Azucarera, 42 $36^{\prime} \mathrm{N}, 5^{\circ} 34^{\prime} \mathrm{O}, 838 \mathrm{~m}$, 13.IX.2007, $10^{\top}$, M. C. Delgado (UL). Madrid, El Escorial, Abantos, Fuente de la Teja, 3.VII.2007, $10^{\pi}$ sobre Abies sp., 24.VII.2007, $10^{\pi}$ sobre Salix $s p$. . V. J. Monserrat (VM), Fuente del Cervunal, 3.VII.2007, $50^{\pi} 0^{\pi}, 4$ 우, 24 . VII.2007, $110^{\pi} 0^{\pi}, 7$ 우 sobre Pinus silvestris, V. J. Monserrat (VM). Valencia, Ademuz, El Rato, Castielfabid, 26.III.2004, $20^{\top} 0^{\top}, 1$ \% en trampa adhesiva con feromona sexual de Matsucoccus feytaudi sobre Pinus pinaster, M. E. Rodrigo (VM), 7.IV.2004, $20^{\top} 0^{\top}, 3$ 우 $ᄋ$ en trampa adhesiva con feromona sexual de Matsucoccus feytaudi sobre Pinus pinaster, M. E. Rodrigo (VM), Chelva, Lomas del Chinchel, 15.III.2004, 3 우 우 en trampa adhesiva con feromona sexual de Matsucoccus feytaudi sobre Pinus pinaster, M. E. Rodrigo (VM).

\section{Hemerobius simulans Walker, 1853}

Especie Holártica, mayoritariamente asociada a coníferas.

Material estudiado: FRANCIA: Haute Garone, Luchon, 26.VI-13.VII.1933, 10", M. E. Mosely (NHM), Sant Béat, 14.VII-18.VIII.1933, $60^{7} 0^{\pi}, 5$ 우, M. E. Mosely (NHM).

\section{Hemerobius gilvus Stein, 1863}

Elemento mediterráneo septentrional, probablemente holomediterráneo, generalmente asociado a planifolios del género Quercus en medios húmedos.

Material estudiado: ESPAÑA: Guipúzcoa, Aiako Harria P. N., Irún, Xaguborda, 30TWN9994, 475 m, 9.VIII.2005, $10^{\pi}$ sobre Quercus pyrenaica, S. Pagola (VM).

\section{Hemerobius atrifrons McLachlan, 1868}

Elemento citado del centro y norte de Europa, generalmente asociado a coníferas del género Larix en medios montanos.

Material estudiado: SUIZA: Grisons, Sertigthal, Upper Engadine, 26.VI-11.VIII.1927, $10^{\pi}$, Mosely (NHM).

\section{Hemerobius eatoni Morton, 1906}

Especie endémica de Canarias (Tenerife, La Palma, Gran Canaria, El Hierro, La Gomera), generalmente asociada a coníferas (Pinus canariensis).

Material estudiado: ESPAÑA: Canarias, Gran Canaria, Agaete, Berrasales, 20.X.1931, 2 웅․ La Palma, Cumbre Vieja, 19.II.1998, 1 ㅇ․ P. Oromí (ULL), Tijarafe, 1.II.1975, 1 ㅇ A. Machado (ULL). Tenerife, Agua García, 10.V.1927, 1 o (MNCNM), Barranco de Tahodio, 19.III.1927,
1 ㅇ (MNCNM), El Médano, 24.III.1932, $10^{\pi}$, IX.1933, 2 우, III.1934, $10^{7}$ (MNCNM), Esperanza, Fuente Fría, 20.X.1927, 1 우 (MNCNM), Esperanza, Hoya de las Raíces, 14.X.1928, 1 웅 "en los pinos", 30.X.1928, $10^{\text {" }}$ (MNCNM), Güimar, 27.V.1928, $10^{\pi}$ (MNCNM), La Esperanza, Laguneta Alta, 2.X.1927, $10^{\pi}$ (MNCNM), La Laguna, 2.XII.1909, 2 우 (identificadas como Sympherobius fallax), 17.X.1920, 1 우, 28.I.1922, 1 우, 12.III.1925, 1 ㅇ , 6.XI.1925, 1 ᄋ , 10.III.1927, 1 ejemplar, 2.V.1927, 1 우, 6.V.1927, 1 ㅇ, 6.VI.1927, 1 우, 11.VI.1927, 1 우, 15.VI.1927, 1 \% , 22.VI.1927, $10^{7}, 3$. VII.1927, $10^{7}$, 7.X.1927, $10^{7}$, 24.X.1927, 1 ㅇ , III.1935, $10^{7}$, (MNCNM), Médano, Los Calderones, 29-30.III.1934, $10^{\pi}, 4$ 우 으 (MNCNM), Mesa de la Mota, 8.III.1910, $10^{\pi}$ (identificado como Stenolomus cabrerai) (MNCNM), Monte de Los Silos,1929, $10^{7}$ (VM), Orotava, Los Órganos, 4.IV.1927, $10^{\pi}$ (MNCNM), Tacoronte, II.1921, $10^{\pi}, 1 \mathrm{f}$ (MNCNM), San Diego, 16.V.1981, 1 오, A. Machado (ULL), Realejo Bajo, VIII.1976, 1 \& , A. Machado (ULL), Santa Úrsula, 12.V.2002, 1m, P. Oromí (ULL), 19.V.2002, 1 오, P. Oromí (ULL), Sauzal, 7.IV.1935, 3 ᄋ $~(\mathrm{MNCNM})$. El material de Canarias del MNCNM muy probablemente Cabrera leg.

\section{Hemerobius nairobicus Navás, 1910}

Especie conocida de Kenia, Uganda, Tanzania, Zaire, Zimbabwe, Lesotho, República de Sudáfrica, Ruanda, Etiopía y Archipiélago de Comores, también citada en Yemen. De biología poco conocida, con cierta tendencia orófila y mayoritariamente capturada a la luz en zonas con abundante vegetación.

Material estudiado: ZIMBABWE: Mashonaland East, Marondera, Marandellas, 1 -31.X.1972, $10^{7}$, P. Ginn (FSCA), XI-XII.1972, $10^{7}$, P. Ginn (FSCA).

\section{Hemerobius reconditus Navás, 1914}

Especie afrotropical conocida del África subsahariana y S. O. de la Península Arábica.

Material estudiado: KENIA: Nairobi, Muguga, 10.VIII.1992，5mm, 5 우, I. González (VM), Nairobi, 13.VIII.1992, $10^{7}, 1$ \& , I. González (VM), 29.VI.1997, $10^{7}$, V. J. Monserrat (VM). Western Province, Mt. Elgon National Park, 2.740 m, 20.I.1992, $20^{7} 0^{7}$ sobre Arundinaria alpina, O. Merkl (HNHM), Chepnalil Cave, 2.500 m, 24-28.I.1992, $10^{7}$, 1 오, O. Merkl (VM), $50^{\top} \sigma^{\top}, 1$ 우, O. Merkl (HNHM), Kimothon River, 3.200 m, 11.I.1992, $20^{\top} \sigma^{\top}, 1$ ㅇ , O. Merkl (VM), $150^{\top} \sigma^{\top}$, 5 우, O. Merkl (HNHM).

\section{Hemerobius bolivari Banks, 1910}

Especie frecuente y ampliamente distribuida por la Región Neotropical desde el sur de México a Tierra del Fuego. De morfología y pigmentación relativamente variable y muy eurioica en la selección de hábitat y sustrato vegetal.

Material estudiado: BRASIL: Sao Paolo, Cravinhos, Fazenda Palmares, $21^{\circ} 18^{\prime} 54^{\prime \prime} \mathrm{S} / 47^{\circ} 47^{\prime} 39^{\prime \prime} \mathrm{O}, 26 . X .2005,1$, 30.VI.2005, $20^{7} \sigma^{7}, 13$.VII.2005, $10^{7}, 28$.VII.2005, $1 \sigma^{7}$, 1.VIII.2005, $10^{\pi}, \quad$ 5.VIII.2005, $10^{\pi}, \quad$ 8.VIII.2005, $10^{\pi}$, 17.VIII.2005, $10^{7}, 19$. VIII.2005, $10^{7}, 27$. VIII.2005, $10^{7}$, 31.VIII.2005, 1 ᄋ , 12.IX.2005, $10^{\top}$, 24.X.2005, $10^{\pi}$, 10.I.2006, 
$10^{\pi}$, 24.I.2006, $10^{\pi}$, de ellos 9 ejemplares a la luz, 7 sobre Coffea arabica, Rogéria Inês Rosa Lara (MZSP).

\section{Hemerobius subacutus (Nakahara, 1966)}

Especie citada de Japón (Islas Ryukyu), Taiwán, Vietnam, Indonesia (Sulawesi) y con reservas de China. La citamos ahora de Nepal e India. La morfología de los ejemplares ahora estudiados concuerda con lo descrito para esta especie por Nakahara (1966) y Makarkin (1993).

Las características de morfología externa, venación y pigmentación alar (Fig. 1) y genitalia de esta especie la hacen inconfundible. Citada de Vietnam por Makarkin (1993) y con el nuevo material ahora aportado, también de Vietnam, consideramos que es el momento de poner en solfa a Hemerobius cercodes Navás, 1917, una de las muchas especies descritas por este problemático autor, y cuya posición taxonómica está aún sin resolver.

Esta especie fue muy probablemente descrita a partir de un único ejemplar de la antigua Indochina (Tonkin, Quang Ien), hoy día Vietnam (Hai Phong, Quang Yen) y su Serie Tipo (ejemplar Tipo o Sintipos, de haber existido varios ejemplares) permaneció en su colección particular (Coll. m.) y sin duda se destruyó durante la Guerra Civil Española y demás vicisitudes sufridas por la colección de este autor (Monserrat, 1985, 1986). Por este motivo fue considerada como nomen dubium por Monserrat (2001).

Los únicos datos que se conocen sobre esta especie (Navás,1917, 1924), por muy paradójicos y sorprendentes que parezcan, no dejan de ser habituales en lo que fue el quehacer de este autor, ya que -con siete años de diferencia- describe dos veces la misma especie, empleando el mismo nombre, el mismo material original, el mismo texto y la misma personal "tipificación". La descripción original (Navás, 1917) es, lógicamente prioritaria, y ésta es repetida en un texto recopilatorio más general (Navás, 1924) donde vuelve a utilizar el mismo material original y a tratarla como especie nueva, describiéndola, "tipificándola" y citándola como n. sp., en vez de mencionarla adecuadamente como especie previamente descrita y conocida.

En lo que respecta a los datos sobre su morfología, pigmentación y genitalia que se desprenden de estas dos únicas referencias (Navás, 1917, 1924) no cabe ninguna duda que se trata de la misma especie que Hemerobius subacutus (Nakahara, 1966). Siguiendo el Artículo 75 del Código Internacional de Nomenclatura Zoológica (ICZN, 1985) y su recomendación $75 \mathrm{E}$, proponemos designar como neotipo de Hemerobius cercodes Navás, 1917 al nuevo ejemplar de Vietnam (Fig. 1) ahora citado en el material estudiado (recolectado a escasos kilómetros de la localidad tipo original) $\mathrm{y}$, en base a esto, proponemos una nueva sinonimia, ya sugerida por Monserrat (2001).

Hemerobius cercodes Navás, 1917: 14

= Hemerobius cercodes Navás, 1924: 139

= Hemerobius subacutus (Nakahara, 1966) n. sin.

= Mesohemerobius subacutus Nakahara, 1966: 202

Material estudiado: INDIA: Assam (¿), Khasis, s.f., 1 ㅇ + 1 ejemplar, McLachlan (NHM). NEPAL: Kathmandú, Kathmandú, 20.V.1983, 1 ㅇ, A. Brendell (NHM). VIETNAM: Yen Bai, Yen Bai, Phù Yên, 21 42'19'N, 10452'32'”E, 40 m, 11.X.2007, 1 우 $\left(\mathrm{A}^{*}\right)$ sobre vegetación arbórea, Neotipo de Hemerobius cercodes Navás, 1917, V. J. Monserrat (VM).

\section{Wesmaelius nervosus (Fabricius, 1793)}

Especie Holártica, de tendencia euroica y orófila.

MATERIAl estudiado: REINO UNIDO: Scotland, Rannoch, 1-15.VII.1903, 1 ejemplar, K. J. Morton (MZB).

Wesmaelius subnebulosus (Stephens, 1836)

Especie Holártica, probablemente introducida en Norte América y sin duda en Nueva Zelanda. Significativamente eurioica.

Material estudiado: ESPAÑA: Almería, Cueva de Sorbes, 1.V.2007, 1 ㅇ sobre Olea europaea, V. J. Monserrat (VM), La Serena, Bedar, 2.V.2007, $10^{7}$ sobre Rhamnus alaternus, 1 ㅇ sobre Ceratonia siliqua, V. J. Monserrat (VM), Puerto de Santa María de Nieva, 1.085 m, 5.V.2007, 1 9 , V. J. Monserrat (VM). Ávila, Embalse de Burguillo, Barraco, 12.V.2007, 1 ㅇ sobre Juniperys oxycedrus, V. J. Monserrat (VM). Cádiz, Pinsapar de Grazalema, 3.V.2005, 1 \% sobre Arbutus unedo, V. J. Monserrat (VM). Canarias, Tenerife, Realejo Bajo, VIII.1976, $10^{7}$, A. Machado (ULL). Granada, La Herradura, 7.IV.2007, 2 우 우 a la luz, V. J. Monserrat (VM). Guipúzcoa, Aiako Harria P.N., Arbiun, Erroiarri, 350 m, 30TXN0094, 23.VI-11.VII.2006, 1 ㅇ en trampa de vino + cerveza sobre Quercus pyrenaica, S. Pagola (VM), Kampazar, 500-650 m, 30TWN4071, 18.VI.2005, 1 o sobre Quercus ilex, S. Pagola (VM), Kausoro, Ibañari, 700 m, 30TWN9588, 27.V.2006, 1 o sobre Acer pseudoplatanus, S. Pagola (VM), Irún, Mt. Jaizkibel, 410 m, 14.VI.2006, 1ㅇ, P. Duelli (PD), Oieleku, Oiartzun, Aiako Harria P. N., 30TWN9589, 500-650 m, 1331.V.2006, 1 ㅇ, S. Pagola (VM), en trampa Kaila en hayedo, 522.VI.2006, $10^{\pi}, 1$ ㅇ sobre Fagus sylvatica, S. Pagola (TAMU), 14.VI.2006, 1 \% , S. Pagola (VM), 6-29.VII.2006, 2mm, 2ff en trampa de vino + cerveza en hayedo, S. Pagola (VM). Madrid, Ciudad Universitaria, 3.III.2005, $10^{7}$ a la luz, V. J. Monserrat (VM), El Escorial, Abantos, Fuente del Cervunal, 24.VII.2007, $10^{7}$ sobre Salix sp., V. J. Monserrat (VM), Arboreto Luis Ceballos, 8.VII.2007, 1 ㅇ sobre Quercus rotundifolia, 24.VII.2007, 1 ㅇ a la luz, V. J. Monserrat (VM), Hoyo de Manzanares, 11.VII.2005, 1 우 sobre Juniperus oxycedrus, V. J. Monserrat (VM), Rivas Vaciamadrid, 26.VI.2000, 1m a la luz, J. I. López (VM). Vizcaya, Larruskain, 106 m, 17.VI.2006, 1f, P. Duelli (PD). Navarra, Portillo de Eraice, 1.537 m, 18.VI.2006, $10^{7}, 3$ 우 우, P. Duelli (PD). FRANCIA: Pyrénées-Atlantiques, Lourdes, Col d'Aubisque, 

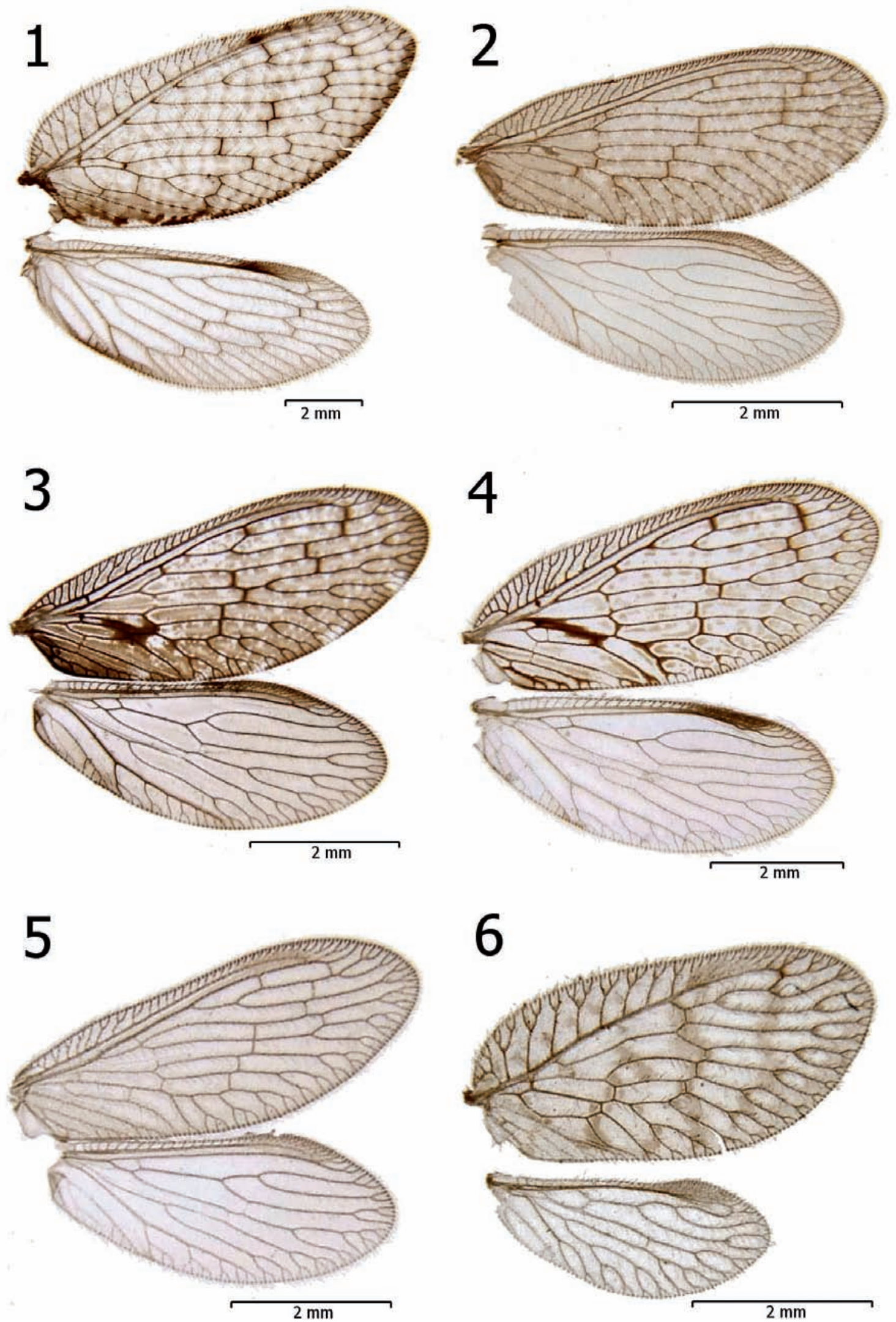
1.390 m, 19.VI.2006, 1 ㅇ, P. Duelli (PD). PORTUGAL: Madeira, Madera, IX.1908, 1 ㅇ leg.? (MZB).

Wesmaelius quadrifasciatus (Reuter, 1894)

Especie Paleártica. Mayoritariamente orófila y asociada a coníferas.

Material estudiado: FRANCIA: Haute Savoie, Chamonix, Mt. Blanc, 18-26.VII.1925, 1 \% , Mosely (NHM). Pyrénées Orientales, Mt. Louis, 22.VI-2.VII.1923, $10^{7}$, Mosely (NHM). Vosges, Gerardmer, 27.V-7.VII.1930, 1 \% Mosely (NHM), Retournermer, 7-31.VII.1930, 4 ○ $\odot$, Mosely (NHM).

Wesmaelius navasi (Andreu, 1911)

Especie asociada a medios xéricos que limitan meridionalmente la Región Paleártica occidental y central, desde Madeira y Canarias a Afganistán, Pakistán y Mongolia y zonas de influencia Afrotropical de Arabia Saudita, Yemen y Omán. De biología poco conocida, mayoritariamente capturada a la luz.

Material estudiado: ESPAÑA: Almería, El Molino de La Serena, Bedar, 1.V.2007, 10 $10^{7}, 2$ $\%$ a la luz, V. J. Monserrat (VM). Canarias, Tenerife, Tenerife, 1902, 1 ejemplar (MZB),

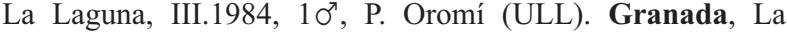
Herradura, 7.IV.2007, $10^{\text {T }}$ a la luz, V. J. Monserrat (VM).

El ejemplar del MZB está identificado por L. Navás como Boriomyia nervosa $\mathrm{F}$. = Wesmaelius nervosus (Fabricius, 1793) y a él debe corresponder la cita de esta especie en Tenerife (Navás, 1906).

Wesmaelius malladai (Navás, 1925)

Especie extramediterránea, orófila y conocida de Europa y de zonas montañosas del Cáucaso y Asia Menor. De biología poco conocida, parece estar asociada a coníferas.

Material estudiado: NORUEGA: Setesdal, Valle, 2.VIII.1980, $10^{7}$, K. R. Tuck (NHM), 4.VIII.1980, $10^{7}$, K. R. Tuck (NHM).

Wesmaelius pinincolus (Ohm, 1967)

Especie orófila endémica de Tenerife, muy escasamente citada.

Material ESTUdiado: ESPAÑA: Islas Canarias, Tenerife, Fuente de la Cañada de la Grieta, 21.X.1927, 1 ㅇ, Cabrera (MNCNM).

\section{Sympherobiinae Comstock}

Sympherobius elegans (Stephens, 1836)

Especie eurioica ampliamente distribuida en Europa y extendida hasta Anatolia y Kazajstán.

Material estudiado: ESPAÑA: Álava, Laiaza, 725 m, 30TWN2814, 5.VII.2005, 1 우 sobre Quercus ilex, S. Pagola (VM), Lalastra, 910 m, 30TVN8146, 5.VII.2005, 1 우 sobre Pinus sylvestris, S. Pagola (VM), Ribera, 750-800 m, 30TVN8144, 12.VII.2005, 1 \& sobre Pinus sylvestris, S. Pagola (VM). Castellón: Insectario de Almazora, 30.IV.2008, $20^{7} 0^{\pi}, 2$ 우 predando sobre Phanococcus citri, V. Petit (IA). Guipúzcoa, Lareo, 750 m, 30TWN7259, 16.VI.2005, 1 ㅇ sobre Corylus avellana, S. Pagola (VM). Madrid, Ciudad

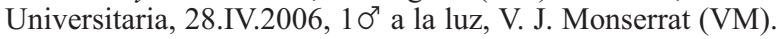

\section{Sympherobius pygmaeus (Rambur, 1842)}

Especie de distribución holomediterránea expansiva desde Canarias hasta Azerbaiján y Kazajstán, marcadamente eurioica.

Material estudiado: ESPAÑA: Álava, Askartza, Trebiñu, 600 m, 30TWN23, 13.VI.2004, 1ㅇ, S. Pagola (VM), Lantziego, El Chital, 500 m, 30TWN3911, 24.V.2004, $10^{7}$, S. Pagola (VM). Ávila, Navalperal de Pinares, 28.VII.2007, $10^{7}$, 1 o sobre Quercus pyrenaica, V. J. Monserrat (VM). León: León, Azucarera, 42³6’ N, 534'O, 838 m, 12-26.IX.2007, $10^{\circ}$ en trampa de vino, M. C. Delgado (VM). Madrid, El Escorial, Abantos, Fuente del Cervunal, 24.VII.2007, $10^{\text {t }}$ sobre Pinus silvestris, V. J. Monserrat (VM), Montarco, Rivas Vaciamadrid,

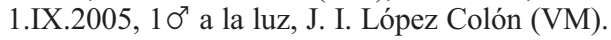

\section{Sympherobius amiculus (Fitch, 1855)}

Especie conocida del S. E. de Canadá (Quebec) y del este, sur y centro de Estados Unidos. Su biología está mayoritariamente asociada a coníferas del género Pinus, también citada sobre Prunus, Quercus y Vitis.

Material estudiado: ESTADOS UNIDOS: California, Julian, 24.V.1974, 1 ㅇ, H. \& M. Townes (FSCA). Florida, Alacahua Co., Gainesville, Doyle Corner Building, 210.VII.1987, 1 ㅇ en trampa de Malaise, P. Skelley (FSCA), Brevard Co., Merritt Isl., 14.IV.1964, 1 ㅇ en trampa de Steiner, G. V. Desin (FSCA), Escambia Co., Warrington, 26-27.V.1963, $10^{\pi}, 1$ ㅇ a la luz, V. F. Grant (FSCA), $10^{\pi}$ a la luz, V. F. Grant (VM), Lake Co., Astor, 10.V.1956, 1 ㅇ sobre Taxodium distichum, F. W. Mead (FSCA), Polk Co., Eaton Park, 17.V.1963, 1 ㅇ en trampa de Steiner, R. E. Vild (FSCA), Seminole Co., Sanford, 28.VI.1966, 1 \% en trampa de Steiner, G. W. Desin

Figs. 1-6.- Alas de: 1) Neotipo de Hemerobius cercodes Navás, 1917, 2) Sympherobius distinctus Carpenter, 1940, 3) S. similis Carpenter, 1940, ejemplar de Panamá, 4) Ídem, de Costa Rica, 5) S. limbus Carpenter, 1940, 6) Paratipo de Notiobiella jaimei n. sp. Escala en mm.

Figs. 1-6.- Wings of: 1) Neotype of Hemerobius cercodes Navás, 1917, 2) Sympherobius distinctus Carpenter, 1940, 3) S. similis Carpenter, 1940, specimen from Panama, 4) Ditto, from Costa Rica, 5) S. limbus Carpenter, 1940, 6) Paratype of Notiobiella jaimei n. sp. Graphic scale in $\mathrm{mm}$. 
(FSCA). Illinois, Karnak, 23.VI.1932, $10^{7}, 1$ \& , Ross, Doziev \& Park (INHS), S. Joseph, 25.V.1913, $10^{\pi}, 12$.VI.1913, 1 \%, H. Lawson (INHS), Peoria, s.f., $10^{\pi}$, F. F. Frison (INHS), Urbana, 27.VI.1927, 10, Frisson \& Ross (INHS), IX.1932, $10^{7}$, T. H. Frison (INHS). Maryland, Montgomery Co., Bethesda, 9.VII.1984, 10, H. V. Weemes (VM). New Mexico, Hatch, 29.VIII.1974, 1우, H. \& M. Townes (FSCA). Oklahoma, Latimer Co., VI.1986, 1 ㅇ, VII.1986, 1 오 X.1986, 1 우, VI.1987, 1 ㅇ, K. Stephan (FSCA), III.1989, $10^{7}$, K. Stephan (VM), IV.1989, 1 ㅇ, K. Stephan (FSCA), V.1989, 1 ㅇ, K. Stephan (FSCA). Tennessee, Gatlinburg, GSMNP, 3500', S.W. Sweeps, 19.VI.1947, 1 ᄋ, 2.VII.1947, 1 ㅇ, R. H. Whittaker (INHS).

\section{Sympherobius occidentalis (Fitch, 1855)}

Especie conocida del E. y S. E. de Estados Unidos y Norte de México. Su biología está mayoritariamente asociada a coníferas del género Pinus. Los abundantes granos de polen de pinos en el interior del tubo digestivo de alguno de los ejemplares ahora estudiados no solo corrobora esta asociación, sino que introduce nuevos elementos sobre la alimentación de sus imagos. El aspecto de su genitalia masculina externa se anota en la figura 11.

MATERIAL eStudiado: ESTADOS UNIDOS: Florida, Marlon Co., Ocala National Forest, Sharpe's Ferry, 31.III.1976, 1 ejemplar en trampa de Malaise, J. R. Wiley (FSCA). Illinois, Urbana, 14.VII.1920, 1 \& H. R. Lawson (INHS), Vienna, 5.VI.1951, $10^{7}$, Ross \& Rich (VM). Kentucky, Marshall Co., 2.IX.1984, 1 ㅇ, P. Skelley (FSCA). Oklahoma, Latimer Co., IV.1987, $10^{\pi}\left(0^{7 *}\right)$, K. Stephan (VM).

Sympherobius perparvus (McLachlan, 1869)

Especie conocida del centro y oeste de Estados Unidos, Canadá (Alberta, British Columbia y Saskatchewan) y norte de México, de biología poco conocida. El grado de curvatura y desarrollo del proceso caudal del ectoprocto en los machos es relativamente variable y se anota en la figura 10 .

Material estudiado: ESTADOS UNIDOS: Arizona, Cochise Co., Westside Willcox Dry Lake, nr. Cochise, 4.200', 2.IX.1991, $20^{7} 0^{\pi}$, R. Miller \& L. Stange (VM). California, Stanislaus Co., Turlock, 1.VI.1972, $10^{7}$, L. A. Lacey (FSCA). Nebrasca, Valentine Refuge, 5.VI.1972, $10^{7}\left(0^{7 *}\right)$, H. \& M. Townes (FSCA), 1m, H. \& M. Townes (VM), 6.VI.1972, $10^{7}$, H. \& M. Townes (FSCA).

\section{Sympherobius barberi (Banks, 1903)}

Especie ampliamente conocida de Estados Unidos y Méjico, citada puntualmente de Canadá (Ontario), introducida en Bermudas y de la que existen citas de los Archipiélagos de Revillagigedo, Hawai, Galápagos y también de Perú, zonas donde ha debido ser introducida. Parece ser una especie eurioica, capturada sobre una gran variedad de sustratos vegetales (Quercus, Eupatorium, Gillarida,
Juniperus, Pseudotsuga, Lantana, Platyopuntia, Asparagus, Mangifera, Taxodium, Solanum, etc.) y, obviamente, a la luz.

MATERIAL ESTUdiado: ESTADOS UNIDOS: Arizona, Portal, 15.VIII.1974, 1, H. \& M. Townes (FSCA). California, Julian, 6.V.1974, $10^{\pi}$, H.\& M. Townes (FSCA), Roverside Co., Riverside, 3.X.1973, 2 우, Lacey (FSCA). Hawaii, Pohakuloa, $6.500 \mathrm{ft}$, 5.IX.1961, 1 \&, H. Townes (VM). Illinois, Ashley, 7.VIII.1917, $10^{x}$, H. Lawson (INHS), Cairo, 15.VII.1937, 1 \& , Burks \& Mohr (INHS), Elizabeth, 7.VII.1917, 1 ㅇ , H. Lawson (INHS), Fairfield, 14.VII.1948, 1m, Mills \& Ross (INHS), Maredosia, 22.VIII.1917, 2 ㅇ ㅇ, H. Lawson (INHS), Urbana, 10.VIII.1932, 1 o sobre Quercus macrocarpa, H. H. Knight (INHS). Michigan, Rudyard, 4.VI.1957, 1 ej., H. Townes (FSCA). Texas, Fredericksburg, 11.V.1988, 1 ㅇ, H. \& M. Townes (FSCA), 17.V.1988, 2 우, H. \& M. Townes (FSCA), San Patricio Co., Sinton, Welder Wildlife Refuge, 29-30.V.1980, 1 \&, G. B. Fairchild (FSCA).

Sympherobius umbratus (Banks, 1903)

Especie conocida de la zona central del tercio oriental y occidental de Estados Unidos (Arizona, Arkansas, Georgia, Missouri, North Carolina, Oklahoma, Pennsylvania, South Carolina, Virginia) y con reservas de New Mexico (Oswald, 1988) y la cita más meridional en Florida que ahora se anota hace más factible la veracidad de esta cita.

MATERIAL ESTUdiado: ESTADOS UNIDOS: Florida, Alachua Co., Gainesville, Doyle Corner Building, 12.V.1978, 1 o a la luz, F. W. Mead (VM). Oklahoma, Latimer Co., VIII.1986, 1 \& en trampa de Malaise, K. Stephan (FSCA).

Sympherobius angustus (Banks, 1904)

Especie conocida desde S. O. de Canadá (British Columbia), O. de Estados Unidos y México (Durango, México D.F., Nuevo León, Oaxaca, Coahuila, Guanajuato, Jalisco, Michoacán). De carácter montano y generalmente asociada a coníferas (Picea). La cita de Costa Rica dada por Monserrat (1998) se comenta al hablar de Sympherobius similis.

Se trata de una especie de morfología muy variable que ha generado varias sinonimias (Oswald, 1988). Corroborando este hecho, hemos estudiado numerosos ejemplares con una gran variabilidad en su coloración y venación alar, solapándose con otras especies conocidas, especialmente del grupo de Sympherobius angustus según Oswald, 1988 (Sympherobius angustus, S. quadricuspis, S. bifasciatus, S. californicus y $S$. constrictus), alguna de ellas conocida en base a escasos ejemplares y / o que no han sido citadas de nuevo desde la descripción original.

Hemos observado, tanto en el material anteriormente estudiado (Monserrat, 1990b, 1998, 2004), como en el material ahora anotado, muy diversa 
pigmentación tegumentaria, antenal y alar. También respecto a la venación hemos estudiado ejemplares con evidentes "irregularidades" en su venación, desde un número variable de $\mathrm{Sr}$, a carecer de bifurcación en el $1^{\circ} \mathrm{Sr}$ de las alas anteriores y ser independiente a $\mathrm{Ma}$, a carecer de venilla proximal entre $\mathrm{R}_{1}-\mathrm{R}_{4+5}$ en las alas anteriores e incluso carecer de venilla proximal entre $R_{1}-R_{4+5}$ en una de las alas anteriores y poseer hasta tres venillas en el otro ala.

En este margen de coloración y variabilidad alar (tres Sr en alas anteriores) entran los pocos caracteres no generalistas o sin interés taxonómico que fueron dados en la descripción original de Sympherobius pupillus Navás, 1915, especie que fue muy probablemente descrita a partir de un único ejemplar de México: San José, "Tamps" (Tamaulipas), de la que no hay nuevos datos desde su descripción original y cuya Serie Tipo (ejemplar Tipo o Sintipos, de haber existido varios ejemplares) permaneció en su colección particular (Coll. $m$.) y sin duda se destruyó durante la Guerra Civil Española y demás vicisitudes sufridas por la colección de este autor (Monserrat, 1985, 1986). Por este motivo fue considerada como nomen dubium por Monserrat (1990a, b).

Pocos datos definitorios pueden recabarse de la descripción de esta especie (Navás, 1915), y aunque parece fundamentar la validez de esta especie en la presencia de tres sectores del radio en las alas anteriores, en la "discusión" anota: "Un ejemplar muy defectuoso. Se trata probablemente de un Sympherobius con tres sectores radiales, pero al estar el ala anterior incompleta no se puede asegurar. De todas formas considero que la descripción y el esquema son suficientes para diferenciar la especie". La presencia de venación anómala con tres sectores del radio en Sympherobius angustus ha sido anteriormente indicada y en algunos ejemplares la venilla proximal entre $R_{1}-R_{4+5}$ es tan corta que parece que la vena $R_{4+5}$ sale directamente de $R$, en cualquier caso las figuras que da para la genitalia masculina son completamente irrelevantes.

A partir de nuevo material recolectado, es el momento de resolver definitivamente la situación taxonómica de esta especie proponiendo un neotipo en base a un ejemplar que se ajusta a los datos de la descripción original, que ha sido recolectado en las proximidades de la Localidad Tipo (según se anota en el material estudiado) y que pertenece a la especie que tratamos, ampliamente conocida de México. Tras ello, se propone una nueva sinonimia:

Sympherobius angustus (Banks, 1904)

= Hemerobius angustus Banks, 1904: 102

= Sympherobius pupillus Navás, 1915: 151 n. sin.
Ya Oswald (1988) en su revisión de las especies neárticas de este género anotaba la escasez de material y la dificultad en la separación de las especies de este grupo en base a caracteres y pigmentación alar, indicando una gran variabilidad y solapamientos entre ellas según los ejemplares y conminando al uso de la genitalia, especialmente interna de los $\mathrm{mm}$ (parámeros), con lo que la identificación de muchos ejemplares, especialmente de las ff en este grupo de especies es harto problemática y subjetiva.

Respecto a la genitalia externa de los mm coincidimos con Klimaszewski \& Kevan (1992) sobre la presencia de cuatro procesos caudales en el ectoprocto de los machos (Fig. 12) y no de una simple "prominencia dorso medial" que anota Oswald (1988) y, en cualquier caso, estos procesos caudales son de desarrollo y grado de curvatura muy variable y este cuarto proceso parece estar más desarrollado en los ejemplares más septentrionales y menos en los más meridionales.

Tampoco la genitalia interna ofrece elementos que se mantengan constantes, o al menos correlacionados con la morfología y pigmentación externas. Así hemos encontrado, tanto en el material anteriormente estudiado (Monserrat, 1990b, 1998, 2004) como en el material ahora anotado, ejemplares dentro de un gradiente de difícil adjudicación a una $u$ otra especie y ejemplares intermedios entre los extremos que anota Oswald (1988) para diferenciar las especies de este grupo, especialmente entre Sympherobius angustus y Sympherobius quadricuspis y hemos hallado ejemplares con lóbulos laterales y caudales de sus parámeros redondeados en su margen caudal (carácter de $S$. angustus) pero con incisión media entre los lóbulos caudales e incisiones profundas en $\mathrm{U} / \mathrm{V}$ entre los lóbulos caudales y los laterales y con presencia de lóbulos anteriores en sus parámeros (caracteres de $S$. quadricuspis) y pequeños dentículos caudales (ausentes en $S$. angustus) que no llegan, ni con mucho, al aspecto que Oswald (1988) anota como definitorio en S. quadricuspis, pareciéndose mucho más a los que se consideran característicos de otras especies como Sympherobius constrictus Oswald, 1988 (que solo posee tres procesos en el ectoprocto de los machos).

Todos estos hechos unen la notable variabilidad en algunos caracteres externos de pigmentación y especialmente de venación alar (habitualmente usados como primeros pasos en las claves existentes) con ciertos caracteres de la genitalia externa también muy variables, como hemos visto, y de la genitalia interna (cuyo ángulo de observación hace además variar enormemente los resultados) que están basa- 
dos en estructuras membranosas (Oswald, 1988) y cuyo grado de esclerificación muy probablemente varíen en función de la edad y del historial sexual de cada ejemplar (al margen de la variabilidad entre las poblaciones), haciendo tediosa y subjetiva (o imposible) la identificación de numerosos ejemplares neárticos de este género, hecho agravado para las hembras.

Tampoco existen suficientes datos discriminatorios sobre su biología para ayudarnos a su diferenciación, ya que algunas de ellas (Sympherobius angustus, S. constrictus, S. bifasciatus y S. californicus) parecen estar asociadas a coníferas (Picea, Cupressus, Pinus), y dentro de este grupo se han citado sobre especies exóticas y cultivadas (Citrus) que poco nos aportan sobre su biología.

Tampoco ayuda, por cierto, su distribución geográfica que pueda resultarnos geográficamente discriminatoria, ya que poseen una distribución muy similar, en ocasiones simpátrida, que abarca desde la British Columbia canadiense y oeste de EE.UU. hasta el N. de México. El hecho de que alguno de los ejemplares de este grupo de especies que ahora se citan (o se han citado) sean simpátridos corrobora esta hipótesis.

Desde nuestro punto de vista no tiene sentido mantener como válidas a especies cuyos caracteres diagnósticos muestran una gran variabilidad, que se solapan y que no se correlacionan de forma constante con otros caracteres externos más o menos difinitorios. Por todo ello cabe suponer la existencia de una gran variabilidad dentro del grupo de especies de Sympherobius angustus (según Oswald, 1988), grupo de especies entre las que hallamos varias especies descritas que representan los extremos conocidos dentro de una serie de caracteres morfológicos muy variables a la que pertenece la mayoría de los ejemplares, que no alcanza uno u otro extremo, y por ello no pueden identificarse con seguridad y es muy probable que especies como Sympherobius angustus (Banks, 1904), S. quadricuspis Oswald, 1988, S. constrictus Oswald, 1988, e incluso $S$. californicus Banks, 1905 no sean más que variaciones poblacionales de una misma especie.

Material estudiado: CANAdÁ: British Columbia, Stone Mt. Pk., $3.800 \mathrm{ft}, 23$.VIII.1973, $10^{\pi}\left(\sigma^{7} *\right)(\mathrm{VM})$. ESTADOS UNIDOS: Idaho, nr. Stanley, 8.VIII.1978, 2 ᄋ, H. \& M. Townes (FSCA). California, Julian, 7.V.1974, 1 ㅇ, H. \& M. Townes (FSCA), 24.V.1974, 1 우 (forma stangei), H. \& M. Townes (VM), Lake Wohlford, 24.IV.1974, 2 우우, H. \& M. Townes (FSCA), 30.IV.1974, 10, H. \& M. Townes (FSCA). Oregón, Corvallis, 6.VI.1978, 1 , H.\& M. Townes (FSCA). MÉXICO: Tamaulipas, Jiménez, 12.V.1999, $1 \mathrm{~m}$ en el interior de una casa, Neotipo de Sympherobius pupillus Navás, 1915, J. Suárez (VM).
Sympherobius californicus Banks, 1905

Especie conocida del occidente del S. O. de Canadá, O. de Estados Unidos y N. O. de México. De biología poco conocida, habiéndose citado sobre Citrus.

Como se ha citado en la especie anterior, la variabilidad en la pigmentación alar anotada para esta especie por Oswald (1988) se corrobora en los nuevos ejemplares ahora estudiados. Esta variabilidad pigmentaria parece estar generalizada en esta especie y en otras especies americanas de este género (ver otras especies ahora citadas, Fig. 2-5 y Oswald, 1988), como lo está en otras especies paleárticas, y es evidente en el grupo de Sympherobius elegans (Stephens, 1836) / Sympherobius pygmaeus (Rambur, 1842), haciendo a veces muy subjetiva la identificación de los ejemplares en base a la morfología y pigmentación alar.

Como hemos visto en la especie anterior, es muy probable que otras especies holárticas, consideradas histórica- / actualmente como válidas, no sean más que variaciones poblacionales o individuales de una misma especie y que, a pesar del esfuerzo realizado en mantenerlas como válidas, nuevas sinonimias serán propuestas cuando series de un mayor número de ejemplares sean estudiadas. De hecho, llama la atención que en faunas similares y análogas como son la Neártica y la Paleártica Occidental (Europea) exista una enorme diferencia en el número de especies de este género (20 Neárticas y 9 Paleárticas Occidentales-Europeas) según Oswald (1988) y Aspöck et al. (2001) y es muy probable que Sympherobius angustus (Banks, 1904) y Sympherobius californicus Banks, 1905 no sean más que formas poblacionales del mismo taxa.

MATERIAL ESTUdiAdO: ESTADOS UNIDOS: California, Julian, 7.V.1974, $10^{\pi}, 8$. V.1974, $10^{7}$, H.\& M. Townes (FSCA), Lake Wohlford, 30.IV.1974, 10", H. \& M. Townes (FSCA).

Sympherobius gratiosus Navás, 1908

Especie ibérica, de posición taxonómica discutida y que ha sido recientemente considerada como una posible especie válida (Aspöck et al., 2001). Hasta que no se revise comparativamente un mayor número de ejemplares, mantenemos su validez como especie por su especial pigmentación alar y su marcada asociación con chopos y álamos (Monserrat, 1980a, 1986, 1994, 2004, Marín \& Monserrat, 1995, Monserrat \& Marín, 1996, etc.). Los nuevos datos que se aportan siguen corroborando esta asociación con Populus spp.

Material estudiado: ESPAÑA: Madrid, Cuelgamuros, Arroyo Guatel Primero, 9.VI.2007, 1 \% sobre Populus piramidalis, 
V. J. Monserrat (VM), Rivas Vaciamadrid, Montarco, 21.V.2007, 1 ㅇ sobre Populus piramidalis, J. I. López Colón (VM).

Sympherobius fallax Navás, 1908

Especie de morfología bastante variable y de distribución circum-sahariana expansiva siendo conocida de las zonas xéricas meridionales del Paleártico Occidental desde la Macaronesia a Pakistán y desde el sur de la Región Afrotropical a Arabia Saudí y Yemen. Su biología poco conocida, asociada a medios xéricos, ha sido mayoritariamente capturada a la luz, también ocasionalmente sobre Ceratonia, Ficus, Persea, Salix, Solanum, Psidium, Albizzia, Citrus, Hibiscus o gramíneas.

Material estudiado: ESPAÑA: Cádiz, Conil, 15.IV.2003, 1 \& a la luz, V. J. Monserrat (VM). Tenerife, La Esperanza, Laguneta Alta, 2.X.1927, 1 ejemplar (MNCNM), La Laguna, 6.X.1928, 1 ㅇ (MNCNM), 5.X.1930, 1 우 a la luz (MNCNM), 19.XI.1930, 1 ejemplar (MNCNM), Monte de Los Silos,1929, $10^{\prime \prime}$ (VM). El material de Canarias muy probablemente Cabrera leg.

\section{Sympherobius gayi Navás, 1910}

Especie conocida de Chile, Argentina, Uruguay, Paraguay, Brasil y Perú, que ha sido introducida como agente de control en Nigeria y accidentalmente, por acción humana, en Portugal e Isla de Pascua (Monserrat, 1991, 1989, Monserrat \& Deretsky, 1999). La citamos por primera vez para la fauna de Bolivia.

Material estudiado: ARGENTINA: Chubut, Pampa Salamarca, $650 \mathrm{~m}, 13$. II.1970, 2 우, M. Gentili (FSCA). BOLIVIA: Cochabamba, Cochabamba, 5.III.1981, $20^{7} 0^{7}$ a la luz, D. Foster (FSCA, VM). CHILE: Coquimbo, Elquí, Las Mercedes, V.1953, $10^{\pi}$, Segovia (FSCA). Santiago, Santiago, $20 \mathrm{~km}$ W. Colina, 15.XI.1968, $30^{\top} \sigma^{\top}$, C. W. \& L. B. O'Brien (FSCA). URUGUAY: Montevideo, Montevideo, II-III.1967, 1 ㅇ, E. Palerm (FSCA).

Sympherobius arizonicus Banks, 1911

Especie conocida del oeste de Canadá (British Columbia), centro y sur-oeste de Estados Unidos, México (Chiapas, Michoacán, Morelos, Oaxaca, Nuevo León, Puebla, Sonora, Veracruz, Baja California) y Costa Rica. De biología poco conocida y mayoritariamente asociada a Cupressus. El aspecto externo de su genitalia masculina se anota en la figura 9.

Material estudiado: ESTADOS UNIDOS: Arizona, Portal, 28.VIII.1974, 1 ㅇ, H. \& M. Townes (FSCA). California, Borrego Sprs., 5.V.1974, $10^{\pi}$, H. \& M. Townes (FSCA), San Bernardino Co., 7.IX.1991, $10^{7}$, L. Stange \& R. Miller (FSCA), San Diego Co., Portero, 8.IV.1974, 107, H. \& M. Townes (VM). New Mexico, Las Cruces, 24.VII.1964, 1 온, Haris (FSCA). Texas, The Basin, Chisos Mts., Big Bend National Park, 13.VIII.1962, $10^{\pi}\left(0^{*}\right)$ a la luz, H. V. Weems (FSCA).
MÉXICO: Baja California, El Sargento, 27.VI.1983, $10^{7}$ a la luz, R. Miller \& L. Stange (VM). Puebla, $3 \mathrm{mi}$ E. Izucar de Matamoras, 20.IV.1962, $10^{\pi}$, F. D. Parquer \& L. A. Stange (VM).

\section{Sympherobius riudori Navás, 1915}

Elemento ibérico muy escasamente citado y de biología casi desconocida. Ha sido capturado a la luz, sobre Juniperus oxicedrus, Pinus halepensis, $P$. nigra, Quercus ilex, y en madera y suelo de Fagus sylvatica en descomposición.

Material estudiado: ESPAÑA: Cádiz, Conil, 15.IV.2003, $10^{\text {T }}$ a la luz, V. J. Monserrat (VM).

\section{Sympherobius miranda Navás, 1908}

Especie conocida de Argentina, Uruguay y Brasil, de biología casi desconocida.

Material estudiado: URUGUAY: Montevideo, Montevideo, II-III.1967, 1 ㅇ, E. Palerm (FSCA).

\section{Sympherobius similis Carpenter, 1940}

Especie conocida de Estados Unidos (Arizona), México (Chiapas, Michoacán, Morelos, Nuevo León, Veracruz), Costa Rica y Panamá, que ahora citamos de Colombia y Perú.

Los ejemplares estudiados mantienen marcada variabilidad de la pigmentación y venación alar ya anotada por Monserrat (1998) que se solapa con lo acontecido en otras especies y que ahora se incrementa al ampliarse su área de distribución y todos los ejemplares estudiados poseen la región basal de Sc, R y Mp muy pálida en las alas anteriores (Fig. $3,4)$. El ectoprocto de la genitalia masculina de los ejemplares estudiados posee tres procesos caudales (Fig. 8) que corroboran los datos anotados para esta especie por Monserrat (1998) y consecuentemente las diferencias objetivas entre la genitalia masculina de esta especie y la de Sympherobius distinctus Carpenter, 1940 quedan muy minimizadas. El ejemplar de Perú es significativamente de menor tamaño y su tegumento es mucho más melánico / pardo, pero no posee diferencias significativas en su genitalia. Los ejemplares portaban abundantes fragmentos de pequeños artrópodos en el interior de su tubo digestivo.

Tras redefinir la genitalia masculina (Fig. 8) y haber corroborado la de Sympherobius angustus (Banks, 1904) (Fig. 12) debe indicarse que a esta especie pertenecen los ejemplares citados por Monserrat (1998) como Sympherobius angustus de Costa Rica.

Material estudiado: COLOMBIA: Valle: Río Jamundi, entre Cali y Jamundi, 1000 m, 27.VIII.1971, 1 오, leg.? (FSCA). 
COSTA RICA: San José, Escazú, 21.V.1987, $10^{\pi}$, H. \& M. Jownes (VM), San Juan (de Lajas de) Zapote, Republic Tobacco Co., 5-7.VII.1964, $30^{\pi} \sigma^{\pi}$, 10ff a la luz, Woodruff (FSCA), $10^{*}\left(\mathrm{~m}^{*}, \mathrm{~A}^{*}\right), 2$ 우 우 a la luz, Woodruff (VM). PANAMÁ: Chiriqui, Bambito, Volcán Baru, 5.200-5.800', 1617.VII.1981, 1 ㅇ * (A*), M. V. Weems \& G. B. Edwards (VM). PERÚ: Lima, Cupiche, 10 km N. Chosica, 25.VI-2.VII.1974, $10^{\pi}$, C. Porter \& L. Stange (FSCA).

\section{Sympherobius limbus Carpenter, 1940}

Especie conocida de Estados Unidos (Texas, Arizona, New Mexico), muy poco citada.

Los ejemplares que hemos estudiado coinciden en lo que respecta a pigmentación general del tegumento y a la genitalia masculina, tanto externa como interna (Oswald, 1988). No así en la pigmentación alar, que es mucho más uniforme, con venas y membrana sin manchas o zonas irregulares más pardas y especialmente en la venación de las alas anteriores de algunos ejemplares. La mayoría de ellos carecen de venillas proximal entre $R_{1}-R_{4+5}$ o distal entre $R_{4+5}$ $\mathrm{R}_{2+3}$, como es habitual en esta especie (Oswald, 1988), pero otros ejemplares presentan vena proximal o distal en una de las alas y no en la otra, e incluso dos venas proximales en una de las alas y no en la otra (Fig. 5). Por todo ello cabe esperar también una marcada variabilidad en la morfología y pigmentación alar de esta especie, esta última ya anotada por Oswald (1988). También la genitalia masculina posee cierta variabilidad en el grado de desarrollo y curvatura de los procesos caudales del ectoprocto, especialmente el infero-ventral (Fig. 7).

Material estudiado: ESTADOS UNIDOS: California, Julian, 12.V.1974, 40 $0^{7} 0^{\pi}$, H.M. \& G.C. Townes (FSCA), $10^{7}$ $\left(0^{7 *}, A^{*}\right)$, H.M. \& G.C. Townes (VM), 7.V.1974, 1 우, H.\& M. Townes (FSCA).

Sympherobius distinctus Carpenter, 1940

Especie conocida de Estados Unidos (Colorado, Arizona), muy poco citada, que ahora citamos para México.

Como en el caso de la especie anterior, los ejemplares que hemos estudiado coinciden en lo que respecta a la pigmentación general del tegumento y de las alas y a la genitalia masculina, tanto externa como interna (Oswald, 1988). No así en la venación pues presentan venilla proximal entre $R_{1}-R_{4+5}$ en ambas alas anteriores o en una de ellas (Fig. 2), hecho que no es característico en esta especie (Oswald, 1988). Por ello cabe esperar una marcada variabilidad en la morfología alar de este carácter en esta especie.

Material ESTUDiAdo: ESTADOS UNIDOS: Arizona, Chiricahua, Mts. Rustler's Park, 27.VII.1987, $10^{7 *}\left(\mathrm{~A}^{*}\right), 1$ \%,
G. H. Nelson (FSCA). MEXICO: Guerrero, 6.4 mi S.W. Filo de Caballo, 9000 ft, 8.VII.1987, 1 ㅇ, R. Wharton (VM).

Sympherobius klapaleki Zeleny, 1963

Especie mediterránea septentrional, poco conocida y escasamente citada.

MATERIAl ESTUdiAdO: ESPAÑA: Navarra, Loiti Mendatea, Izko, Ibargoiti, 770 m, 30TXN3124, 1 ㅇ sobre Quercus faginea, S. Pagola (VM).

Nomerobius psychodoides (Blanchard in Gay, 1851)

Especie ampliamente citada de Argentina, Chile, Perú, Brasil y Uruguay.

Alguno de los ejemplares estudiados portaba abundantes setas y fragmentos de pequeños artrópodos en el interior de su tubo digestivo, coincidiendo con lo anotado por Monserrat (1998, 2003).

Material estudiado: ARGENTINA: Chubut, Comodoro Rivadavia, Puerto Visser, $5 \mathrm{~m}, 18 . \mathrm{II} .1970,1$ \% , M.Gentili (FSCA), Pampa Salamarca, 650 m, 13.II.1970, 1 \&, M. Gentili (FSCA). Neuquén, Bajada Marucho, 10.II.1970, 1 \&, M. Gentili (VM), Zapala, 1.600 m, 12.XII.1969, 1 우, leg.? (FSCA), 2 우, leg.? (VM).

\section{Nomerobius signatus (Hagen, 1861)}

Especie conocida de Chile, Argentina y Perú. Su biología es poco conocida, citada a la luz o en trampas de Malaise y sobre Austrocedrus chilensis (Cupresaceae).

Material estudiado: ARGENTINA: Chubut, Pampa Salamarca, 650 m, 13.II.1970, 2 우, M. Gentili (FSCA). CHILE: Coquimbo, Elquí, Las Mercedes, V.1953, 1 , Segovia (VM).

Nomerobius spinosus Oswald, 1990

Especie conocida de Chile y Argentina y de biología casi desconocida. Ha sido mayoritariamente capturada a la luz en medios xéricos, en ocasiones hasta $3.100 \mathrm{~m}$.

Material estudiado: ARGENTINA: Río Negro-Neuquén, Arroyo Malahuaca - Río Limai, 15.I.1971, 1 오, M. Gentili (VM). Río Negro, Pilcaniyeu, 7.XI.1974, 1 ㅇ, M. Gentili (FSCA).

Nomerobius cuspidatus Oswald, 1990

Especie conocida de Argentina, Bolivia y Chile. $\mathrm{Su}$ biología es poco conocida, citada a la luz y sobre Tillandsia usneoides (Bromeliaceae) y Tamarix gallica (Tamaricaceae), Monserrat (2003) aporta datos sobre su biología y estadios juveniles. Alguno de los ejemplares estudiados portaban en el interior del tubo digestivo setas, escamas y restos de artrópodos (junto a esporas, polen e hifas de 

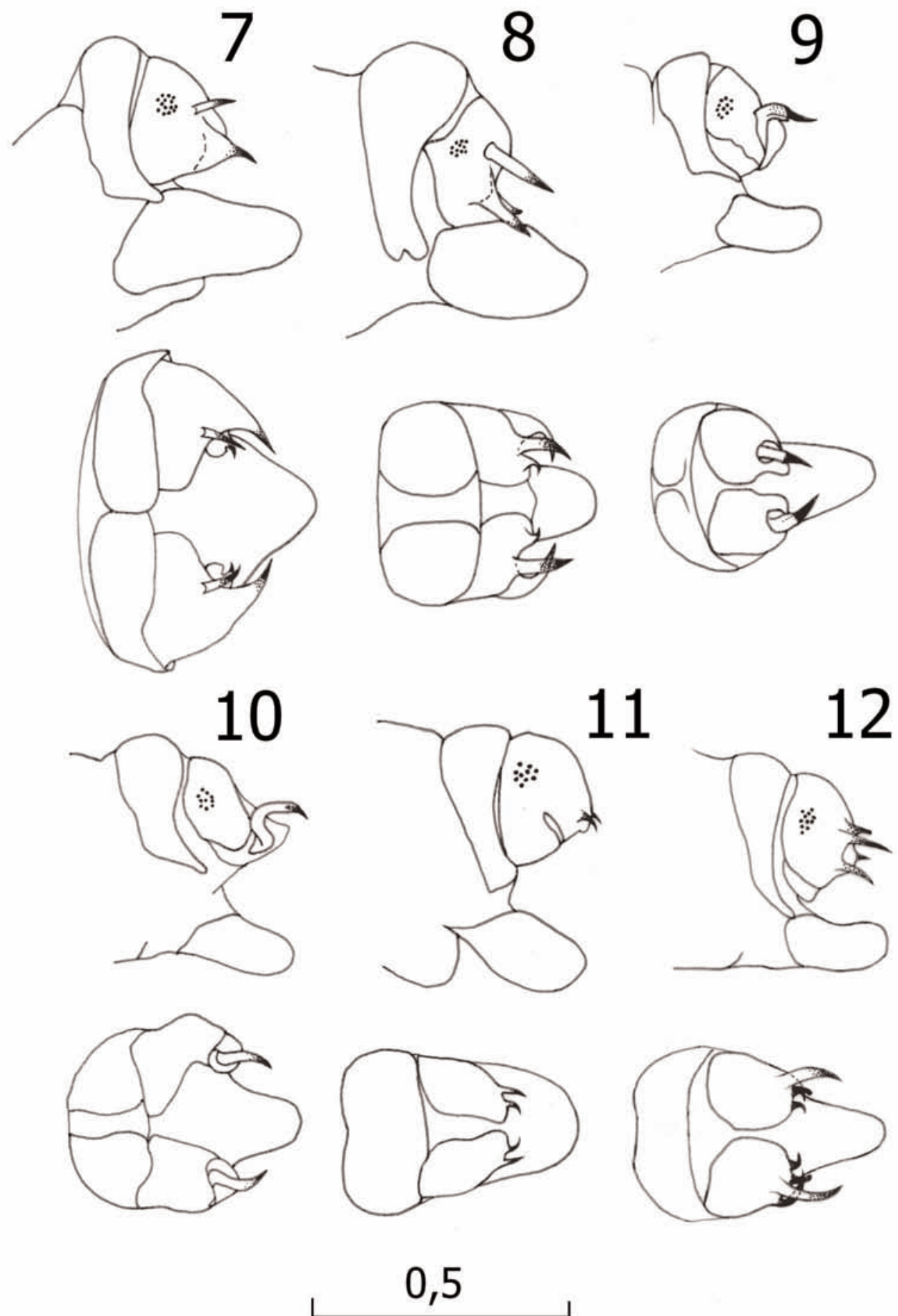

Figs. 7-12.- Esquema de extremo abdominal del macho (omitidas estructuras internas), vista lateral y dorsal, de diferentes especies de Sympherobius. 7) S. limbus, 8) S. similis, 9) S. arizonicus, 10) S. perparvus, 11) S. occidentalis, 12) S. angustus. Escala en $\mathrm{mm}$ para todas las figuras.

Figs. 7-12. - Scheme of male abdominal apex (internal structures omitted) in lateral and dorsal view of different species of Sympherobius. 7) S. limbus, 8) S. similis, 9) S. arizonicus, 10) S. perparvus, 11) S. occidentalis, 12) S. angustus. Graphic scale in $\mathrm{mm}$ for all figures. 
hongos) corroborando el carácter depredador de esta especie (Monserrat, 2003).

Material estudiado: ARGENTINA: Neuquén, San Martín de los Andes, 18.X.1970, 10 $0^{7}$, M. Gentili (FSCA), 10.XI.1970, 1 \& , M. Gentili (VM). BOLIVIA: Cochabamba, Cochabamba, 5.III.1981, 3 우우 a la luz, D. Foster (FSCA), 2 우 이 la luz, D. Foster (VM).

\section{Notiobiellinae Nakahara}

\section{Notiobiella jaimei n. sp.}

Material estudiado: TIPO: VIETNAM: An Giang, Chau Doc, Nui Sam, 1040'34"N, 10504'39'E, $150 \mathrm{~m}$, 29.X.2007, $10^{\text {त }}$ sobre Acacia sp., V. J. Monserrat (VM).

PARATIPOS: VIETNAM: An Giang, Chau Doc, Nui Sam,

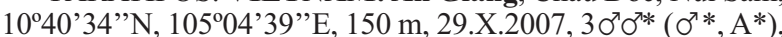
2 ○ $*^{*}\left(\right.$ ( *) sobre Acacia sp., V. J. Monserrat (VM), $10^{\pi}$ sobre Acacia sp., V. J. Monserrat (MNCNM).

DESCRIPCIÓN: Coloración general del tegumento y de las alas amarillo verdosa, muy pálida, salvo las zonas que se indican. Cabeza con región dorsal y ventral al globo ocular más pardas, éste es pardo cobrizo. Antenas de ambos sexos con escapo subcilíndrico y pedicelo fusiforme, flagelómeros pálidos y progresivamente algo más oscuros en el tercio distal. Número de flagelómeros en los $0^{7} \sigma^{7}(\mathrm{n}$ $=1)$ : 47, en las 우우 $(\mathrm{n}=1)$ : 49. Palpos labiales y maxilares pardo pálido con el penúltimo palpómero pardo oscuro. Tórax con pronoto trapecial y con zonas laterales y especialmente el ángulo antero lateral pardo más oscuro. Alas según la figura 6 . Longitud de las alas anteriores de 4,60 $\mathrm{mm}$ en los $0^{7} \sigma^{7}(\mathrm{n}=1)$ y de $4,94 \mathrm{~mm}$ en las 우우 $(\mathrm{n}=1)$. Venación de las alas anteriores de coloración uniforme y membrana con tenues sombras grisáceas más oscuras y especialmente situadas en las bifurcaciones de las venas y a ambos lados de las venillas trasversales (Fig. 6). Pterostigma poco notorio en su coloración. Alas posteriores de $3,23 \mathrm{~mm}$ en los $0^{\pi} \sigma^{\pi}(\mathrm{n}=1)$ y $3,10 \mathrm{~mm}$ en las 우우 $(\mathrm{n}=1)$. Venación y membrana de las alas posteriores de coloración uniforme. Pterostigma expandido pero poco notorio en su coloración (Fig. 6). Patas de coloración uniforme sin manchas o artejos más oscuros. Uñas curvas y no dilatadas en su base. Abdomen de coloración uniforme. Sin otras diferencias apreciables entre sexos.

En los $0^{\pi} 0^{\pi}$ los terguitos y esternitos son trapeciales, más anchos en su margen caudal. El $9^{\circ}$ terguito estrecho, no está interrumpido sobre la línea dorsal media, levemente ensanchado hacia la zona ventral. El $9^{\circ}$ esternito fuertemente convexo. Ectoprocto con proceso caudal fusiforme con abundantes setas, levemente espatulado hacia su extre- mo y arqueado hacia la línea media, su margen interno porta un pequeño proceso digitiforme ventral corto y romo en posición media (Fig. 13-15), callo cercal con 14-19 tricobotrias. Gonarco poco esclerificado, elipsoide en vista lateral (Fig. 18). Entoprocesos recurvados hacia arriba y levemente hacia la línea media, su extremo algo más esclerificado y con dos dentículos terminales (Fig. 16-18). Arceso membranoso, subcilíndrico, armado de pequeños dentículos / setas, levemente arqueado y su extremo caudal es bífido y cada una de sus ramas es portadora de dos grupos de espinas triangulares (Fig. 17-19). Faloningua compleja, formada por membranas replegadas sobre sí y portadoras de pequeños dentículos / setas (Fig. 16-18). Parámeros muy tenues y membranosos, con procesos laterales globosos y margen caudal levemente cóncavo (Fig. 20). Hipandrio triangular (Fig. 21).

En las 우 익 los terguitos y esternitos son trapeciales, más anchos en su margen caudal. El $8^{\circ}$ terguito progresivamente más estrechado hacia la línea media ventral. El $9^{\circ}$ terguito no se fusiona sobre la línea media ventral. Ectoprocto circular, callo cercal con 14-18 tricobotrias. Gonapófisis laterales semiesféricas con estilos pequeños. Placa subgenital rectangular, levemente bilobulada en su margen caudal (Fig. 22).

Etimología: Dedico esta nueva especie al que fue mi querido amigo y compañero Jaime Aguilar, como muestra de agradecimiento a su amistad y su cariño, y como reconocimiento a su fortaleza y su coraje ante la enfermedad que lo llevó a las galaxias y a su esfuerzo ante la intransigencia de los que no acabaron de respetar su otra forma de amar.

DisCUSIÓN: Son relativamente pocas las especies del tropical género Notiobiella que son conocidas de la Región Oriental. En su catálogo mundial Monserrat (1990 a) lista siete especies descritas de la Región Oriental: $N$. viridinervis Banks, 1913 de Sri Lanka, N. valida Banks, 1920 de Indonesia (Borneo), N. gloriosa Navás, 1933 de China, N. ochracea Nakahara, 1966 de Iriomote Island y Formosa, $N$. dentata Monserrat, 1990 de Indonesia (Bali), N. luisae Monserrat, 1990 de Indonesia (Flores, Sumba) y N. tumida (Navás, 1925) nom. dub. de Indonesia (Java), a las que hay que añadir Notiobiella subolivacea Nakahara, 1915 y N. stellata Nakahara, 1966 descritas de Iriomote Island y Japón, pero citadas por Nakahara (1960, 1966) de Formosa, Notiobiella sp. 3 citada por Monserrat (1990b) de Sri Lanka y N. nguyeni Makarkin, 1993 posteriormente descrita de Vietnam. 


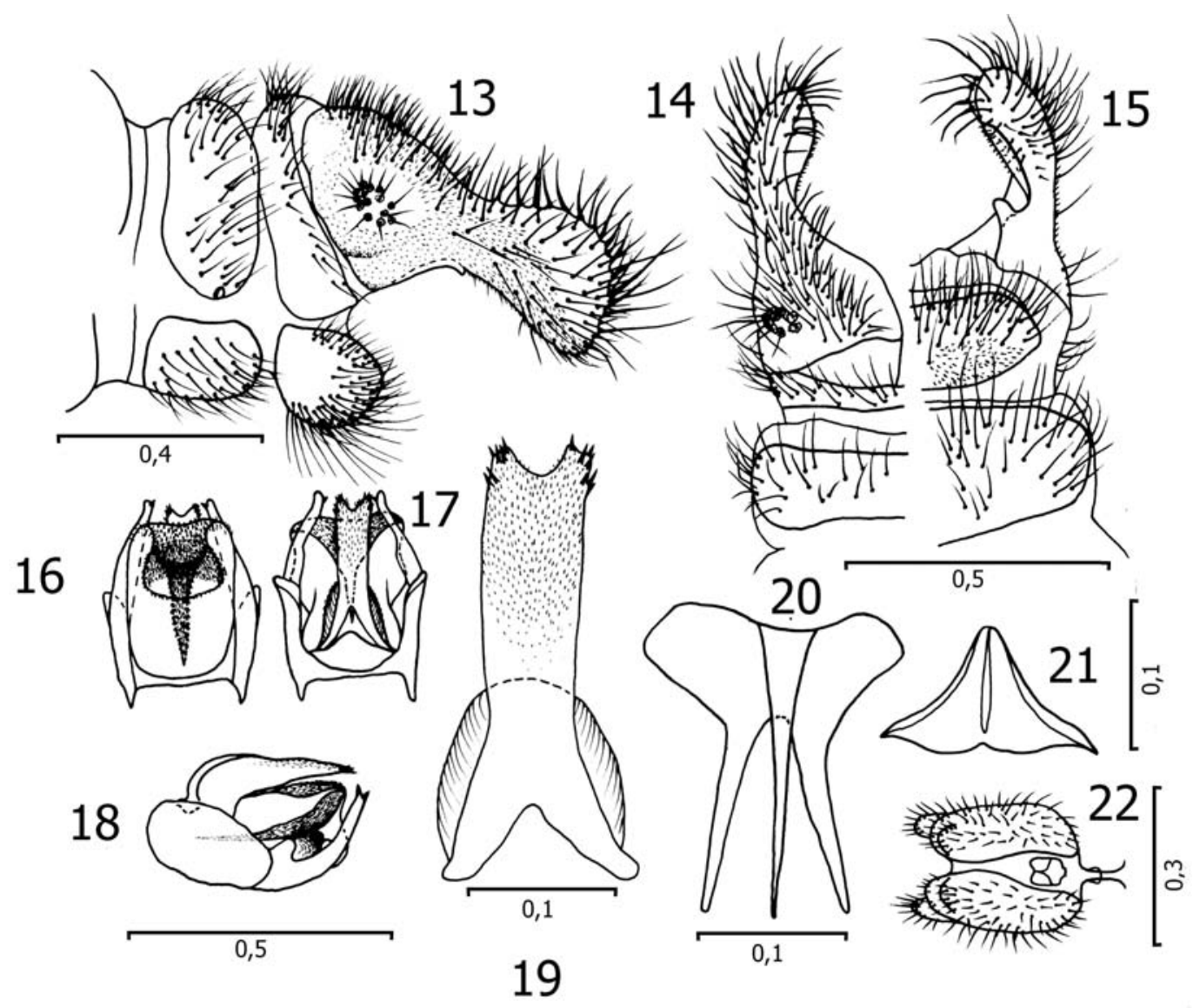

Figs. 13-22.- Notiobiella jaimei n. sp. $0^{7}$. Extremo abdominal, 13) vista lateral, 14) dorsal y 15) ventral. Complejo gonarco-arceso-entoprocesos-falolingua, 16) vista ventral, 17) dorsal y 18) lateral. 19) Arceso, vista dorsal. 20) Parámeros, vista dorsal. 21) Hipandrio, vista ventral. 22) Notiobiella jaimei n. sp. ․ . Extremo abdominal, vista ventral. Escala en mm.

Figs. 13-22.- Notiobiella jaimei n. sp. $\sigma^{7}$. Abdominal apex, 13) lateral view, 14) dorsal view and 15) ventral view. Gonarcus-arcessus-entoprocessus-phalolingua, 16) ventral view, 17) dorsal view and 18) lateral view. 19) Arecessus, dorsal view. 20) Paramera, dorsal view. 21) Hypandrium, ventral view. 22) Notiobiella jaimei n. sp. $ᄋ$. Abdominal apex, ventral view. Graphic scale in mm.

En base a lo que conocemos sobre estas especies, ninguna de ellas presenta una combinación de caracteres similares a lo citado para $N$. jaimei n. sp., ni en lo que respecta a su coloración tegumentaria, ni a su venación y envergadura alar, ni sobre todo a su genitalia masculina o femenina, mostrando por el contrario mayor similitud con otras especies afrotropicales y en especial con $N$. turneri Kimmins,1933 especie citada de la República de Sudáfrica y Zimbabwe, y en particular con N. nitidula Navás, 1910, especie citada de Kenia, Angola, República de Sudáfrica, Senegal, Nigeria, Gambia, Zambia, Madagascar, Mali, Mozambique y Archipiélagos de Comores y de Cabo Verde
(Tjeder, 1961; Monserrat, 1984, 1990b, 1992a, 2004; Monserrat \& Deretsky, 1999; Hölzel, 2007).

Respecto a Notiobiella turneri Kimmins,1933, la genitalia masculina se asemeja a $N$. jaimei $\mathrm{n}$. sp. (Tjeder, 1961; Monserrat \& Deretsky, 1999), pero es muy diferente el grosor, longitud y curvatura del ectoprocto y de su proceso interno (Fig. 13-15) y también son distintos los arcesos, el gonarco, los entoprocesos y los parámeros (Fig. 16-20). Sobre su aspecto externo difieren en su coloración tegumentaria, la anchura y pigmentación de las alas y la ausencia de venilla entre el $1^{\circ} \mathrm{Sr}$ y Ma y la sombra oscura entre $\mathrm{Cu}_{\mathrm{a}}$ y $\mathrm{Cu}_{\mathrm{p}}$, características de $N$. turne$r i$, no existen en $N$. jaimei n. sp. (Fig. 6). 
Respecto a Notiobiella nitidula Navás, 1910, la morfología externa, el aspecto general, tamaño y pigmentación tegumentaria de ambas especies es muy similar aunque $N$. nitidula es notablemente más verdosa que $N$. jaimei $\mathrm{n}$. sp. También la venación y la pigmentación alar de ambas especies es muy afín (Monserrat, 1984, 2004), aunque en $N$. jaimei n. sp., el $\mathrm{SR}_{1}$ está marcadamente bifurcado dos veces en las alas anteriores y el pterostigma de las alas posteriores es mucho más extenso y dilatado y estas alas son proporcionalmente mayores (Fig. 6). Parece existir diferencias en el número de flagelómeros (47-49 en $N$. jaimei n. sp., frente a 39-44 en $N$. nitidula) y sus patas poseen coloración uniforme en $N$. jaimei $\mathrm{n}$. sp., mientras que en $N$. nitidula son algo más oscuros el extremo de las tibias y los tarsos. Respecto a la genitalia masculina, en N. nitidula (Monserrat, 1984, 2004) el ectoprocto es mucho más ancho y triangular, no tan espatulado y delgado (Fig. 13-15) y es diferente y más apical la posición de su proceso interno (Fig. 15). También son diferentes los parámeros (Fig. 20), el entoproceso es más curvo y divergente en $N$. nitidula y su arceso carece de dentículos caudales que son evidentes en $N$. jaimei n. sp. (Fig. 19).

Para acabar la discusión de esta nueva especie un último apunte ha de hacerse sobre una de las especies anteriormente citada: Notiobiella gloriosa Navás, 1933, especie descrita de Fukien (Provincia de Fujian) de China oriental y de la que nada nuevo se sabe, ni ha vuelto a ser citada desde su parca descripción original (Navás, 1933). Por lo poco que puede recabarse de su descripción, parece ser una especie de bastante mayor envergadura que $N$. jaimei $\mathrm{n}$. sp. (ala anterior $6,40 \mathrm{~mm}$, frente a 4,60-4,94 $\mathrm{mm}$ en $N$. jaimei $\mathrm{n}$. sp.), de alas anteriores algo apuntadas en su ápice, con un mayor número de venillas en el campo costal (12 frente a 8 en $N$. jaimei n. sp.) y venas longitudinales tres (y no dos) veces bifurcadas antes de alcanzar el margen alar. La supuesta pigmentación alar tampoco las asemeja y todos estos caracteres mucho más nos recuerdan a las citadas $N$. ochracea Nakahara, 1966 y en especial a N. stellata Nakahara, 1966 descrita de Iriomote Island (Japón) y de la vecina Formosa (Nakahara, 1966), de la que con toda probabilidad es sinónima. Como es habitual en la obra de este autor, no tipifica adecuadamente la especie, ni especifica el número de ejemplares de la Serie Tipo, ni su ubicación, y hasta que no se localice el ejemplar tipo o se recolecte nuevo material para designar un neotipo, esta sinonimia no puede asegurarse.

La especie parece poseer preferencia por la vegetación arbórea, todos los ejemplares se recolectaron sobre acacias. En el interior de las mem- branas peritróficas de los ejemplares se han observado abundantes fragmentos de tegumento, patas y antenas de pequeños artrópodos, principalmente de áfidos, junto a material vegetal diverso, esporas y algún grano de polen.

\section{Psectra diptera (Burmeister, 1839)}

Especie ampliamente distribuida en la Región Paleártica desde Irlanda a Japón y probablemente introducida en Norte América, donde ha sido citada en la zona oriental de Canadá y Estados Unidos y de la que anotamos nuevas citas que sugieren una ya antigua introducción hacia el interior. Mayoritariamente capturada a la luz y sobre gramíneas y otras plantas, herbáceas.

MATERIAL ESTUdiAdo: ESTADOS UNIDOS: Illinois, Illinois, V.1910, 1 \% , leg.? (INHS), Ch. Co. (Christian County?), V.1855, $10^{\pi}$, Roses Swp (VM), Urbana, 24.VII.1891, 1 ㅇ, Hart (INHS).

\section{Megalominae Krüger}

Megalomus hirtus (Linnaeus, 1761)

Especie europea extendida hasta Armenia. De biología poco conocida.

Material estudiado: CHECOSLOVAQUiA: Bohemia, Chodau, s.f., 1 \% , Stein (NHM). FRANCIA: Haute Garone, Luchon, 26.VI-13.VII.1933, 1 , M. E. Mosely (NHM). Pyrénées Orientales, Argeles, Hospitalets, 7-25.VI.1995, $10^{7}, 2$ 우 우, K. Jordan (NHM), Taxo les Pins, 17-29.VI.1984, 2 우, S. \& A. Brooks (NHM), Mount Luois, R. Tet, 22.VI-2.VII.1923, 1 ㅇ, M. E. Mosely (NHM).

\section{Megalomus tortricoides Rambur, 1842}

Especie conocida del Mediterráneo septentrional extendida hacia Armenia y Georgia en el Cáucaso. De biología poco conocida.

Material estudiado: FRAnCia: Pyrénées Orientales, Axat, R. Aude, 20.VI.1923, 1 \& , Mosely (NHM), Vernet, 2328.VI.1899, 1 \%, Wlsm (NHM).

\section{Megalomus pyraloides Rambur, 1842}

Especie Atlanto-mediterránea conocida de España, Francia, Italia y Croacia. De biología poco conocida.

Material estudiado: Material estudiado: FRANCIA: Pyrénées Orientales, Thues les Bains, 7.VII.1900, 1 우, Welsm (NHM).

Megalomus tineoides Rambur, 1842

Elemento holomediterráneo expansivo, de biología poco conocida. 
Material estudiado: ESPAÑA: Almería, El Molino de La Serena, Bédar, 17.VII.2007, $10^{7}, 5$ 우 a la luz, V. J.

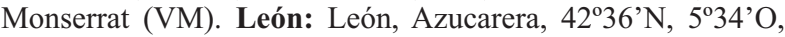
838 m, 13.IX.2007, $10^{7}$, M. C. Delgado (UL). FRANCIA: Córcega, Niolo Region, Upper Golo Valley, VII-VIII.1961, $20^{\pi} 0^{\pi}, 1$ ㅇ , A. Meats (NHM).

\section{Megalomus fidelis (Banks, 1897)}

Especie conocida de la región central y oriental de Estados Unidos y noreste de México, también citada de Canadá (British Columbia, Quebec, Ontario). Aparentemente asociada a coníferas del género Pinus.

MATERIAL ESTUDIADO: ESTADOS UNIDOS: Oklahoma, Latimer Co., V.1987, $10^{7}, 1$ ㅇ, K. Stephan (VM), VI.1987, 2ff, K. Stephan (FSCA), VIII.1987, 1 ㅇ, K. Stephan (FSCA), IX.1987, 10", 1으, K. Stephan (FSCA). South Carolina, Greenville, 26.VIII.1956, 1 ㅇ, L. \& G. Townes (FSCA).

\section{Drepanepteryginae Krüger}

\section{Drepanepteryx phalaenoides (Linnaeus, 1758)}

Especie paleártica, asociada a medios boscosos y húmedos.

Material estudiado: AUSTRIA: Burgenland, Leitha Gebirge, 10-23.VI.1956, 1 오 E. \& I. Clasey (NHM). BULGARIA: Borovetz, Samokov, 18.V.1958, 1 \%, leg.? (NHM). FRANCIA: Haute Garone, Sant Béat, 14.VII-18.VIII.1933, $10^{\pi}, 2$ 우, M. E. Mosely (NHM).

\section{Gayomyia falcata (Blanchard en Gay, 1851)}

Especie conocida de Argentina, Chile, Archipiélago de Juan Fernández y Bolivia, de biología poco conocida. Citada sobre Nothofagus y Laurelia.

Material estudiado: CHILE: X Región, Río Azul, 30.XI.1962, 1 ejemplar, A. Kovacs (NHM).

\section{Microminae Krüger}

Micromus paganus (Linnaeus, 1767)

Especie paleártica, de biología poco conocida.

Material estudiado: NORUEGA: Nordland Fylke, Grönlien, 29.VII.2007, $10^{7}$, J. Fernández (VM).

Micromus variegatus (Fabricius, 1793)

Especie Paleártica que probablemente ha sido introducida desde Japón a la British Columbia Canadiense y que está asociada a medios húmedos.

MATERIAL ESTUdiAdo: ESPAÑA: Guipúzcoa, Oiartzun, Aiako Harria P. N., Aiendola, 100 m, 30TWN9592, 12.V.2003, $10^{7}$, I. Zabalegui (VM), Oieleki, 500-650 m, 30TWN9589, 16.V.2006, $10^{\pi}$ sobre hierba, S. Pagola (VM), 5.VI2006, 1 \& sobre Urtica dioica, S. Pagola (VM), Pillotasoro, $420 \mathrm{~m}$, 30TWN9491, 24.IV.2003, 1 \% , I. Zabalegui (VM), Aralar P. N., Atalegieta, 280 m, 30TWN7366, 25.VI.2004, $10^{7}$, S. Pagola (VM).

\section{Micromus angulatus (Stephens, 1836)}

Elemento Holártico. Su biología es poco conocida, aparentemente eurioica.

MATERIAL ESTUdiado: ESPAÑA: Guipúzcoa, Leizarán, 250 m, 30TWN88, 2.V.2005, 1 ㅇ , I. Zabalegui (VM). Madrid, Rivas-Vaciamadrid, 9.XI.2002, 1 \& , J. I. López Colón (VM). ESTADOS UNIDOS: Minnesota, Eagles N.E. Eagles Nest, 13.VI.1959, $10^{\pi}$, 16.VIII.1959, $10^{\pi}$, W.V. Balduf (INHS).

\section{Micromus timidus Hagen, 1853}

Especie frecuente en la zona intertropical de Asia, África, Australia y Oceanía. Marcadamente eurioica.

Material estudiado: VIETNAM: An Giang, Chau Doc, Nui Sam, 1040'34”'N, 10504'39'”E, 150 m, 29.X.2007, 1 ㅇ sobre Acacia sp. V. J. Monserrat (VM). Lao Cai, Bac Ha, $22^{\circ} 32^{\prime} 25^{\prime \prime} \mathrm{N}, 104^{\circ} 17^{\prime} 43^{\prime \prime} \mathrm{E}, 900 \mathrm{~m}, 6 . X .2007,1$ ㅇ a la luz, V. J. Monserrat (VM). Son La, Co Noi, 2119'38'N, 103'54'33'E, 610 m, 11.X.2007, 1 ㅇ sobre Bambusa bambos (Poaceae) V. J. Monserrat (VM).

\section{Micromus posticus (Walker, 1853)}

Especie conocida de Canadá y mayoritariamente del N. E y E. de Estados Unidos. Citada de zonas abiertas sobre vegetación herbácea y arbustiva.

MATERIAL ESTUDIADO: ESTADOS UNIDOS: Illinois, Algonquin, 8.VIII.1895, $10^{7}$, leg.? (INHS), 16.IX.1904, 1 \& , leg.? (INHS), 4.VII.1907, 10", W. A. Nason (INHS), 24.X.1910, $10^{7}$, W. A. Nason (INHS), Augerville, 13.XI.1928, 1 ㅇ , H. Rutherford (INHS), 6.IV.1929, 10 , H. Rutherford (INHS), Brownfield, 17.VIII.1916, 1 \% , Carp. (INHS), Chicago, 28.IV.1906, 1 \% , Carp (INHS), Danville, 9.X.1907, 10 $0^{\pi}$ Carp (INHS), Dubois, 21.V.1917, 1 \% , leg.? (INHS), 3.VI.1919, 1 ㅇ, leg.?, (INHS), Goreville, 13.V.1932, 1 오, H. L. Dozier (INHS), Havara, 12.VII.1932, $10^{7}$, Dozier \& Park (INHS), Herord, 24.VII.1930, 2 우 ᄋ, Knight \& Ross (INHS), Kansas, 23.VII.1930, 10", Knight \& Ross (INHS), Lawrenceville, 7.IX.1933, 1오, Ross \& Mohr (INHS), Oakwood, 14.VI.1930, $10^{\pi}, 1$ 오, Frison (INHS), 22.VII.1930, 1 오, Hattes \& Tauben (INHS), Urbana, 11.XI.1912, 1 \& , Carp (INHS), White Heath, 29.V.1933, $10^{\pi}$, Knight \& Ross (INHS). Ohio, Prebe Co., Hueston Woods, 2.XI.1978, $10^{\pi}$, R. J. Foss (VM). Oklahoma, Latimer Co., VI.1987, $10^{7}$, K. Stephan (VM). South Carolina, Lemon SWP, $2.5 \mathrm{mi} \mathrm{S}$. Banberg, 1.VIII.1965, 1 ㅇ, F. W. Mead (FSCA).

\section{Micromus subanticus (Walker, 1853)}

Especie holártica extendida al Caribe (República Dominicana, Haití y Cuba) hasta Costa Rica. Se ha hallado sobre diferentes sustratos vegetales tanto arbóreos como herbáceos, generalmente en medios abiertos. 
MATERIAl ESTUdiado: ESTADOS UNIDOS: Illinois, Acadia Parish, Eunice, 6.II.1959, 1ㅇ, Ross \& Stannnard (INHS), Champaing Co., 30.VI.1975, 1 ᄋ , M. E. Farris (INHS), Oakwood, 14.VI.1930, $10^{7}$, Frison (INHS), Volo, 27.VII.1934, 2 우, Delong \& Ross (INHS). Oklahoma, Latimer Co., 5 mi W. Red Oak, VIII.1986, 1 ㅇ, K. H. Stephan (FSCA).

\section{Micromus tasmaniae (Walker, 1860)}

Especie ampliamente citada en la Región Australiana, Australia (especialmente $\mathrm{S}$ y E), Tasmania, Nueva Zelanda (ambas North \& South I. y Stewart I.) e islas adyacentes (Kermadec I., Chatam I., I. Antipodas y Auckland I.). De biología poco conocida, parece estar asociada a vegetación baja en zonas abiertas. A pesar de las sugerencias que se han hecho sobre esta especie y sus posibles poblaciones / subespecies / especies (New, 1988; Wise, 1993, 2000), preferimos mantener el criterio tradicional que se tiene sobre esta variable especie de la que existen numerosas variedades y formas descritas.

Material estudiado: AUSTRAliA: New South Wales, Griffith, X.1967, $20^{7} 0^{\top}, 2$ 우, W. J. Kemp (INHS), Tathra, 20 m, I.1929, 1 \% , leg.? (FSCA). Queensland, Mt. Cootha, V- VII, $10^{\prime \prime}$, leg. ¿ (FSCA). Mt. Glorious, 17.XI, $10^{7}$, leg.? (VM). NUEVA ZELANDA: Bay of Plenty, Rotorua, 12-15.X.1970, 1 \& , FRI, J. Bain (FSCA), 25-29.XII.1970, 1 \& , FRI, J. Bain (FSCA).

\section{Micromus variolosus (Hagen, 1886)}

Especie conocida de la Región Neártica occidental desde la British Columbia, Yukon y Saskatchewan a México. De biología poco conocida.

MATERIAL ESTUdiado: ESTADOS UNIDOS: Colorado, Otero Co., $13 \mathrm{mi} \mathrm{S}$. Cloudcroft, 4.VI.1980, 1 ㅇ, Webb \& Lisowski (INHS).

\section{Micromus numerosus Navás, 1910}

Especie conocida de Japón, Corea del Sur, Taiwán y China, que ahora citamos de Vietnam, extendiendo significativamente su área de distribución conocida. La morfología del ejemplar estudiado coincide con lo descrito para esta especie por Monserrat (1993) y Monserrat \& Deretsky (1999). Los abundantes fragmentos de artrópodos que contenía su tubo digestivo corrobora el carácter depredador de los imagos, ya anotado por Monserrat (2001) y Sato \& Takada (2004).

Material estudiado: VIETNAM: Lai Chau, Pa Tan, $22^{\circ}$ $28^{\prime} 13^{\prime}$ 'N, $103^{\circ} 12^{\prime} 09$ '”, $320 \mathrm{~m}, 9 . X .2007,1$ 우 sobre vegetación de ribera, V. J. Monserrat (VM).

Micromus igorotus Banks, 1920

Especie conocida de Filipinas, Japón (Islas Ryukyu), Taiwán, Tailandia, Indonesia, China y
Malasia, que ahora citamos de Laos. La morfología del ejemplar estudiado coincide con lo descrito para esta especie por Monserrat (1993) y los abundantes fragmentos de artrópodos que contenía su tubo digestivo corrobora su carácter depredador, ya anotado por Monserrat (2001).

Material estudiado: LAOS: Luang Prabang, Luang Prabang, 19'53'23'”N, 102'08'08'E, 310 m, 21.X.2007, 1 ㅇ sobre Bambusa bambos (Poaceae) V. J. Monserrat (VM).

\section{Micromus oblongus Kimmins, 1935}

Especie ampliamente distribuida en la región oriental del centro y sur de África. Mayoritariamente capturada a la luz y trampas de Malaise por lo que su biología es muy poco conocida. Parece tener amplia distribución altitudinal y amplio margen en la elección del sustrato vegetal sobre la que ha sido hallada.

Material estudiado: SUdÁfriCa: Cape Province, Swartbergpas, 9-19.XII.1968, 1 ㅇ, Potgieter \& Jones (TM).

\section{Micromus canariensis Esben-Petersen, 1936}

Especie endémica de las Islas Canarias, de biología poco conocida.

Material estudiado: ESPAÑA: Islas Canarias, Tenerife, Cañada de Diego Hernández, XI.1934, $10^{7}, 2$ ㅇ 우, La Laguna, 6.X.1927, $10^{7}$, San Andrés, 27.IV.1930, 1 ㅇ, Sauzal, 7.IV.1935, 1웅 Tacoronte, 1921, $10^{7}$ (muy probablemente Cabrera leg.) (MNCNM).

\section{AGRADECIMIENTOS}

Deseamos agradecer a las instituciones mencionadas su amabilidad por permitirnos estudiar el material que se ha citado, a Miguel Ángel Alonso Zarazaga por sus opiniones sobre algunas cuestiones de taxonomía y nomenclatura planteadas, y a Eduardo Ruiz y Ana García Moreno por su ayuda en la realización de las fotografías y las láminas.

\section{Referencias}

AsPöck, H., AsPöck, U. \& Hölzel, H., 1980. Die Neuropteren Europas. Goecke \& Evers. Krefeld. t. I 495 pp., t. II 355 pp.

AspöcK, H., HöLzEL, H. \& AspöcK, U., 2001. Kommentierter Katalog der Neuropterida (Insecta: Raphidioptera, Megaloptera, Neuroptera) der Westpaläarktis. Denisia, 2: 5-606.

BANKS, N., 1904. Neuropteroid Insects of New Mexico. Transactions of the American Entomological Society 30: 97-110, pl. I. 
HölzEL, H., 2007. Hemerobiidae of the Afrotropical Region: a review of current knowledge. Annali del Museo civico di Storia Naturale di Ferrara, 8: 73-78.

INTERNATIONAL COMMISSION ON ZOOLOGICAL NOMENClATURE, 1985. International Code of Zoological Nomenclature, $3^{\text {rd }}$ Edition. International Trust for Zoological Nomenclature. London. 338 pp.

Kevan, D. K. McE. \& Klimaszewski, J., 1986. Notes on the Hemerobiidae or brown lacewings flies (Neuroptera) of Canada and Alaska. Neuroptera International, 4: 7-22.

Kevan, D. K. McE. \& Klimaszewski, J., 1987. The Hemerobiidae of Canada and Alaska. Genus Hemerobius L. Giornale Italiano di Entomologia, 16: 305-369.

Klimaszewski, J. \& Kevan, D. K. McE., 1985. The brown lacewing flies of Canada and Alaska (Neuroptera: Hemerobiidae). Part I. The Genus Hemerobius Linnaeus: systematics, bionomics and distribution. Lyman Entomological Museum and Research Laboratory Memoire, 15: iv + 1-119.

Klimaszewski, J. \& Kevan, D. K. McE., 1987a. The brown lacewing flies of Canada and Alaska (Neuroptera; Hemerobiidae). Part II a. The genus Wesmaelius Krüger. Neuroptera International, 4: 153-204.

Klimaszewski, J. \& Kevan, D. K. McE., 1987b. The brown lacewing flies of Canada and Alaska (Neuroptera; Hemerobiidae). Part II b. The genus Wesmaelius Krüger. Neuroptera International, 4: 245-274.

Klimaszewski, J. \& Kevan, D. K. McE., 1988a. New distribution data for Canadian Hemerobiidae (Neuroptera): Genera: Hemerobius Linnaeus, Micromus Rambur and Wesmaelius Kruger. Supplement [1st]. Revue d'Entomology du Québec, 32: 9-15.

Klimaszewski, J. \& Kevan, D. K. McE., 1988b. The brown lacewing flies of Canada and Alaska (Neuroptera: Hemerobiidae). Part III. The genus Micromus Rambur. Giornale Italiano di Entomologia, 19: 31-76.

Klimaszewski, J. \& Kevan, D. K. McE., 1990a. Distribution data for some Nearctic species of the genus Hemerobius Linnaeus and Wesmaelius Krüger (Neuroptera: Hemerobiidae). Supplement 2. Neuroptera International, 5: 205-210.

Klimaszewski, J. \& Kevan, D. K. McE., 1990b. New distribution data for some American species of the genus Hemerobius Linneaus, with a redescription of Hemerobius alpestris Banks (Neuroptera: Hemerobiidae). Annals of the Transvaal Museum, 35: 151-155.

Klimaszewski, J. \& Kevan, D. K. McE., 1990c. The genus Micromus Rambur in North America, with special reference to Canada and Alaska, a synopsis (Insecta: Neuroptera: Hemerobiidae). In: M. W. Mansell and H.
Aspöck (eds.). Advances in Neuropterology. Proceedings of the Third International Symposium on Neuropterology, South African Department of Agricultural Development, Pretoria: 63-66.

Klimaszewski, J. \& Kevan, D. K. McE., 1992. Review of Canadian and Alaskan brown lacewing flies (Neuroptera: Hemerobiidae) with a key to the genera. Part IV: The genera Megalomus Rambur, Boriomyia Banks, Psectra Hagen and Sympherobius Banks. Annals of the Transvaal Museum, 35: 435-457.

MAKARKIN, V. N., 1990. A check-list of the NeuropteraPlanipennia of the USSR far East, with some taxonomic remarks. Acta Zoologica Hungarica, 36: 37-45.

MAKARKIN, V. N., 1993. The brown lacewings from Vietnam (Neuroptera Hemerobiidae). Tropical Zoology, 6: 217-226.

Marín, F. \& Monserrat, V. J., 1995. Contribución al conocimiento de los neurópteros de Zaragoza (Insecta, Neuropteroidea). Zapateri, 5: 109-126.

McEwen, P., New, T. R. \& Whittington, A. E., 2001. Lacewings in the Crop Environment. Cambridge University Press. Cambridge. 546 pp.

Monserrat, V. J., 1980a. Contribución al conocimiento de los neurópteros de Toledo (Neuroptera, Planipennia). Graellsia, 34: 177-193.

Monserrat, V. J., 1980b. Sobre las especies africanas del género Notiobiella Banks, 1909 (Neuroptera, Planipennia, Hemerobiidae) I. Neuroptera International, 1(1): 26-33.

Monserrat, V. J., 1984. Sobre las Especies Africanas del Género Notiobiella Banks, 1909, II. (Neuroptera, Planipennia, Hemerobiidae). In: J. Gepp (ed.). Progress in World's Neuropterology, Proceedings of the $1^{\text {st }}$ International Symposium on Neuropterology, Graz: 99-124.

Monserrat, V. J., 1985. Lista de los tipos de Mecoptera y Neuroptera (Insecta) de la colección L. Navàs, depositados en el Museo de Zoología de Barcelona. Miscellània Zoològica, 9: 233-243.

Monserrat, V. J., 1986a. Sinopsis de los hemeróbidos de la Península Ibérica (Neuroptera, Planipennia, Hemerobiidae). Actas de las VIII Jornadas de la Asociación española de Entomología, Sevilla: 12001223.

Monserrat, V. J., 1986b. Longinos Navás, his neuropterological work and collection. In: J. Gepp, H. Aspöck and H. Hölzel (eds.). Recent Research in Neuropterology. Proceedings of the $2^{\text {nd }}$ International Symposium on Neuropterology, Graz: 173-176.

Monserrat, V. J., 1990a. A Systematic checklist of the Hemerobiidae of the world (Insecta: Neuroptera). In: M. W. Mansell \& H. Aspöck (eds.). Advances in Neuropterology. Proceedings of the Third International Symposium on Neuropterology, Pretoria: 215-262. 
Monserrat, V. J., 1990b. Systematic studies on Hemerobiidae (Insecta: Neuroptera). In: M. W. Mansell \& H. Aspöck (eds.). Advances in Neuropterology. Proceedings of the Third International Symposium on Neuropterology, Pretoria: 67-88.

Monserrat, V. J., 1990c. Some brown lacewings from Equatorial Guinea (Neuroptera, Planipennia: Hemerobiidae). Journal of African Zoology, 104: 1722.

Monserrat, V. J., 1990d. Revisión de la obra de L. Navas, II: El Género Micromus Rambur, 1842 (Neuropteroidea, Planipennia: Hemerobiidae). Graellsia, 46: 175-190.

Monserrat, V. J., 1991. Nuevos datos sobre los hemeróbidos ibéricos (Insecta, Neuroptera: Hemerobiidae). Zoologica Baetica, 2: 101-113.

Monserrat, V. J., 1992a. New data on the Afrotropical brown lacewings (Neuroptera: Hemerobiidae). Journal of the entomological Society of Southern Africa, 55(1): 123-136.

Monserrat, V. J., 1992b. On some African species of the genus Micromus Rambur, 1842 (Insecta: Neuroptera: Hemerobiidae). In: M. Canard, H. Aspöck \& M. W. Mansell (eds.). Proceedings of the Fourth International Symposium on Neuropterology, Toulouse: 271.278.

Monserrat, V. J., 1993. New data on some species of the genus Micromus Rambur, 1842 (Insecta: Neuroptera: Hemerobiidae). Annali del Museo Civico di Storia Naturale "G. Doria”, 89: 477-516.

Monserrat, V. J., 1994. Nuevos datos sobre las especies de hemeróbidos ibéricos (Neuroptera: Hemerobiidae). Boletín de la Asociación española de Entomología, 18(3-4): 9-25.

Monserrat, V. J., 1996. Revision del genero Hemerobius de Latinoamérica (Neuroptera, Hemerobiidae). Fragmenta entomologica, Roma, 27(2): 399-523.

Monserrat, V. J., 1998. Nuevos datos sobre los hemeróbidos de América (Neuroptera, Hemerobiidae). Journal of Neuropterology, 1: 109-153.

Monserrat, V. J., 2000. Revisión del Género Nusalala (Neuroptera, Hemerobiidae). Fragmenta entomologica, Roma, 32(1): 83-162.

Monserrat, V. J., 2001. New data on the Brown Lacewings from Asia (Neuroptera, Hemerobiidae). Journal of Neuropterology, [2000], 3: 61-97.

Monserrat, V. J., 2003. Contribución al conocimiento de los hemeróbidos de Patagonia y Tierra del Fuego (Insecta, Neuroptera: Hemerobiidae). Graellsia, 59(1): 37-56.

Monserrat, V. J., 2004. Nuevos datos sobre algunas especies de hemeróbidos (Insecta: Neuroptera: Hemerobiidae). Heteropterus Revista de Entomología, 4: $1-26$.
Monserrat, V. J. \& Deretsky, Z., 1999. New Faunistical, Taxonomic and Systematic Data on Brown Lacewings (Neuroptera:Hemerobiidae). Journal of Neuropterology, 2: 45-66.

Monserrat, V. J. \& Marín, F., 1996. Plant substrate specificity of Iberian Hemerobiidae (Insecta: Neuroptera). Journal of Natural History, 30: 775-787.

NAKAhara, W., 1960. Systematic studies on the Hemerobiidae (Neuroptera). Mushi, 34(1): 1-69, 16 pl.

NAKAHARA, W., 1966. Hemerobiidae, Sisyridae and Osmylidae of Formosa and Ryukyu Islans (Neuroptera). Kontyû, 34(3): 193-207, lám. 3, 4.

NavÁs, L., 1906. Catálogo descriptivo de los Insectos Neurópteros de las Islas Canarias. Revista de la Real Academia de Ciencias Exactas, Físicas y Naturales de Madrid, 4: 687-706.

NAVÁs, L., 1915. Neue Neuropteren. Entomologische Mitteilungen, 4: 146-153.

NAVÁs, L., 1924. Faune entomologique de l'Indochine francaise. Névroptères de l'Indochine. Opuscules de l'Institut Scientifique de l'Indochine, 8(3): 125-161.

NAVÁs, L., 1929. Insectos Neurópteros del Museo de Hamburgo. Memorias de la Real Sociedad Española de Historia Natural, 15: 315-322.

NAVÁs, L., 1933. Névroptères et insectes voisins - Chine et pays environnants. Notes d'Entomologie Chinoise, 13: $1-10$.

New, T. R., 1988a. A Revision of the Australian Hemerobiidae (Insecta: Neuroptera). Invertebrate Taxonomy, 2: 339-411.

NEw, T. R., 1988b. Hemerobiidae (Insecta: Neuroptera) from New Guinea. Invertebrate Taxonomy, 2: 605-632.

Oswald, J. D., 1988. A Revision of the Genus Sympherobius Banks (Neuroptera: Hemerobiidae) of America north of Mexico with a synonymical list of the world species. Journal of the New York Entomological Society, 96(4): 390-451.

Oswald, J. D., 1990. Revision of the Neotropical Brown Lacewing Genus Nomerobius (Neuroptera: Hemerobiidae). Annals of the Entomological Society of America, 83(1): 18-29.

OSWALD, J. D., 1993a. Revision and cladistic analysis of the world genera of the family Hemerobiidae (Insecta: Neuroptera). Journal of the New York Entomological Society, 101(2): 143-299.

Oswald, J. D., 1993b. A new genus and species of brown lacewing from Venezuela (Neuroptera: Hemerobiidae), with comments on the evolution of the hemerobiid forewing radial vein. Systematic Entomology, 18: 363-370.

Oswald, J. D., 1994. A new phylogenetically basal subfamily of brown lacewings from Chile (Neuroptera: Hemerobiidae). Entomologica scandinavica, 25: 295-302. 
Oswald, J. D., 1996. A new brachypterous Nusalala species from Costa Rica, with coments on the evolution of flightlessness in brown lacewings (Neuroptera: Hemerobiidae). Systematic Entomology, 21: 343-352.

SATO, T. \& TAKADA, H., 2004. Biological studies on three Micromus species in Japan (Neuroptera: Hemerobiidae) to evaluate their potential as biological control agents against aphids: 1 . Thermal effects on development and reproduction. Applied Entomology and Zoology, 39(3): 417-425.

TJEDER, B., 1961. Neuroptera-Planipennia. The lacewings of Southern Africa 4. Family Hemerobiidae. En: B. Hanström, P. Brinck \& G. Rudebec. South African Animal Life, 8: 296-408.

Wise, K. A. J., 1993. Species of Micromus (Neuroptera: Hemerobiidae) in New Zealand. Records of the Auckland Institute and Museum, 30: 93-117.
Wise, K. A. J., 2000. Re-evaluation of three species of Australasian Hemerobiidae (Insecta: Neuroptera). New Zealand Entomologist, 22: 15-21.

Recibido, 13-V-2008

Aceptado, 23-X-2008

Publicado, 29-XII-2008 
